\title{
Adaptive Routing and Recharging Policies for Electric Vehicles
}

\author{
Timothy M. Sweda, Irina S. Dolinskaya, Diego Klabjan \\ Department of Industrial Engineering and Management Sciences \\ Northwestern University \\ 2145 Sheridan Rd. \\ Evanston, Illinois 60208, USA \\ E-mail: tsweda@u.northwestern.edu,dolira@northwestern.edu,d-klabjan@northwestern.edu
}

March 22, 2015

\begin{abstract}
Planning a trip with an electric vehicle requires consideration of both battery dynamics and the availability of charging infrastructure. Recharging costs for an electric vehicle, which increase as the battery's charge level increases, are fundamentally different than refueling costs for conventional vehicles, which do not depend on the amount of fuel already in the tank. Furthermore, the viability of any route requiring recharging is sensitive to the availability of charging stations along the way. In this paper, we study the problem of finding an optimal adaptive routing and recharging policy for an electric vehicle in a network. Each node in the network represents a charging station and has an associated probability of being available at any point in time or occupied by another vehicle. We develop efficient algorithms for finding an optimal a priori routing and recharging policy and then present solution approaches to an adaptive problem that build on a priori policy. We present two heuristic methods for finding adaptive policies - one with adaptive recharging decisions only and another with both adaptive routing and recharging decisions. We then further enhance our solution approaches to a special case of grid network. We conduct numerical experiments to demonstrate the empirical performance of our solutions.
\end{abstract}

Keywords: electric vehicles; adaptive routing; recharging policies; dynamic programming 


\section{Introduction}

Battery electric vehicles (EVs) have become a practical and affordable option in recent years for environmentally conscious drivers looking to reduce their carbon footprint [10]. EVs are powered solely by electricity and recharge by plugging into an outlet or charging station. This feature offers the potential for significant fuel cost savings as well as a host of other benefits, such as fewer greenhouse gas emissions, reduced dependence on foreign oil, and improved power systems management (if vehicle-to-grid services are enabled) [21, 22]. Despite the numerous advantages that EVs have over conventional gasoline-powered vehicles, range anxiety - the worry that an EV's range is insufficient for a driver's commuting needs - remains a chief concern among many potential purchasers [10]. Without the ability to easily recharge away from home, EV owners are restricted primarily to short-distance trips. The expansion of public charging infrastructure to increase opportunities for EV drivers to recharge their vehicles outside their homes has been one popular method for alleviating range anxiety and encouraging greater EV adoption [2]. However, since each charging station can usually only recharge one or two vehicles at a time, and charge times can be on the order of hours, a driver who arrives at a fully occupied station may incur significant inconvenience (e.g., a long wait time) if no other nearby charging station is available. Thus, EV drivers can greatly benefit from taking into account charging station availability and anticipating wait times at the stations while planning their routes, which is the focus of this paper.

We study an optimal EV routing problem in a network where trips may require multiple recharging stops along the way. Since the availability of charging stations is a critical factor in the planning of long-distance routes, an EV driver must not only select which path to take to arrive at the destination as quickly as possible, but also decide where to recharge and what to do in case a desired charging station is unavailable (i.e., wait or seek an alternative station). The uncertainty of charging station availability and wait times within the network, as well as the driver's ability to adaptively make routing and recharging decisions, are unique and critical features of our problem. Furthermore, since the availability of each station may differ, the selection of stopping locations must be part of the routing decision.

In addition to station availability and waiting time, overcharging costs, incurred when an EV's battery is charged near its maximum capacity, are important to consider when creating EV recharging policies for a number of reasons. First, recharging an EV battery while it is already at a high state of charge takes place at a slower rate than when it is more depleted, and storing high levels of charge for prolonged periods of time can shorten the lifespan of the battery. A couple of models describing this relation can be found in [16] and [20]. In addition, overcharging causes battery degradation due to greater stresses from being charged near 
full capacity and excess heat generated during recharging. In the refueling problem for conventional vehicles, the only main disadvantage of traveling with a full tank of fuel is the limited ability to take advantage of lower fuel prices further along the route. Thus, optimal solutions tend to favor filling large quantities and making fewer stops (assuming that stopping costs are considered), but the opposite is often true for optimal EV recharging policies. Therefore, the trade-off among total recharging time, number of charging stops, station availabilities, and wait times must be captured in our EV routing and recharging policy. We consider the problem on a given network with charging stations positioned at every node, each with a corresponding probability of being available and expected waiting time until becoming available (known a priori to the driver). Our goal is to determine an adaptive routing and recharging policy that minimizes the sum of all traveling, waiting, and recharging costs. We assume that whenever the vehicle stops to recharge, it incurs a fixed stopping cost, a charging cost based on the total amount it recharges, and an additional cost when the battery becomes overcharged.

In this paper, we develop and analyze a variety of models depending on the type of decisions to be made and the amount and timing of information available to the EV driver while traveling. In our first model, we consider an optimal adaptive recharging problem for a fixed path, where information about a station's availability is revealed to the driver upon arriving at that location, and the driver decides at that time whether or not to stop. The second model aims to find an optimal adaptive routing and recharging policy for a general network, where path selection is added to the decision space. As the computational complexity of the general network setting makes problem intractable, we develop heuristic solution approaches. First, we find an a priori policy corresponding to an optimal route and charging locations that minimizes the total expected cost. Then, we propose two solution methods for adaptive decision making based on the a priori policies: (1) the driver is committed to a path corresponding to the optimal a priori policy but can change the charging locations along the way; and (2) the driver can adapt both the recharging locations and path in response to the realized availability of charging stations. For the special class of problems with a grid network, we perform an in-depth analysis and further improve the proposed solution methods by taking advantage of the grid structure. A numerical study demonstrates the performance and solution quality of our algorithms using simulated charging infrastructure networks.

This paper is the first in the literature to consider adaptive routing and recharging (or refueling) for range-constrained vehicles. It is also the first to implement two features together that are unique to EVs: overcharging costs and uncertain charging station availability. Thus, the main contributions of this paper are: $(i)$ properties of optimal adaptive and a priori recharging policies that consider EV overcharging char- 
acteristics and uncertain charging station availability; (ii) efficient solution procedures for obtaining a priori and adaptive routing and recharging policies in a network; (iii) models capturing and analyzing various levels of adaptive decision making and information timing; and (iv) an illustrative example and numerical study that establish the value of adaptive decision making in a variety of settings.

The remainder of the paper is organized as follows. Section 2 provides an overview of the existing literature related to EV recharging as it pertains to routing decisions. Section 3 describes the general problem setting and provides a motivating example. In Section 4 we present efficient solution method to find an optimal recharging locations policy for a fixed path. Section 5 analyzes an adaptive routing and recharging policy for a general network. Here, the presented problem complexity motivates heuristic solution approaches that build on a priori routing and recharging policies (also discussed in Section 5). To further improve the proposed solution methods, we study a special class of grid network problems in Section 6. Subsequently, a numerical study is exhibited in Section 7 to demonstrate the performance of the solution algorithms presented in the earlier sections. Finally, Section 8 presents the conclusion of this paper.

\section{Literature review}

The vehicle refueling problem, where a driver must decide at which nodes to refuel as well as how much to refuel in order to minimize the total cost of fuel, has been well studied. It is shown in [9] and [13] that the optimal refueling policy along a fixed path can be solved easily with dynamic programming when fuel prices at each node are static and deterministic. For such a problem, the optimal decision at each node is always one of the following: do not refuel, refuel completely, or refuel just enough to reach the next node where refueling occurs. An algorithm for simultaneously finding the optimal path and refueling policy in a network is detailed in [14], and some combinatorial properties of the optimal policies are explored in [15]. Specifically, it is proven that the problem of finding all-pairs optimal refueling policies reduces to an all-pairs shortest path problem that can be solved in polynomial time. However, all of the aforementioned analyses only consider fuel costs and not stopping or other costs. We include these additional costs in our analysis since they can comprise a significant portion of the total travel cost for an EV and therefore can influence optimal policies.

Several existing models have expanded on the vehicle refueling problem by introducing costs for stopping to refuel and traveling to refueling stations. A generic model for vehicle refueling is presented in [23] that attempts to capture such aspects, penalizing longer routes and routes with more refueling stops. Similar to 
other papers that study the vehicle refueling problem, it assumes that fuel prices at each station are static and deterministic. An analogous model is proposed in [25], which seeks to minimize both the fuel cost and travel distance simultaneously.

Approaches for finding optimal refueling policies when fuel prices are stochastic are presented in [11] and [24]. In [11], a forecasting model for predicting future fuel prices is used to generate parameters for a deterministic mixed integer program, and in [24], a dynamic programming framework is presented that is designed to grant drivers greater autonomy to select the stations where they refuel. These models are difficult to solve analytically, and the authors develop heuristics for obtaining reasonable solutions. In addition, just like the other models of the vehicle refueling problem, these ones do not include any costs that are analogous to battery overcharging costs for EVs, which can be an important component [16, 20]. The authors in [26] address this issue by introducing generalized charging cost functions, however some restrictive assumptions are required in order to perform insightful analysis. A more specific yet tractable overcharging cost function is analyzed in [27], and optimal recharging policies along a fixed path are determined.

To solve the problem of finding a path for an EV within a network with recharging considerations, one thread of research has taken an entirely different approach: having vehicles recharge via regenerative braking rather than by recharging at stations along their paths. As an EV decelerates, it can recapture some of its lost kinetic energy as electrical energy, which can then be used to recharge the battery. It is therefore possible in some cases for an EV's state of charge to increase while traveling rather than decrease, such as when the vehicle is coasting and braking downhill. In [1], the authors model the problem of finding the most energy-efficient path for an EV in a network as a shortest path problem with constraints on the charge level of the vehicle, such that the charge level can never be negative and cannot exceed the maximum charge level of the battery. Edge weights are permitted to be negative to represent energy recapturing from regenerative braking, yet no negative cycles exist. A simple algorithm for solving the problem is provided, and more efficient algorithms are presented in [4] and [19]. It is shown in [4] that the battery capacity constraints can be modeled as cost functions on the edges, and a transformation of the edge cost functions permits the application of Dijkstra's algorithm. The approach described in [19] avoids the use of preprocessing techniques so that edge costs can be calculated dynamically, and it achieves an order of magnitude reduction in the time complexity of the algorithm from [1]. In practice, however, the amount of energy recovered by regenerative braking is insignificant when compared with the amount that must be recharged at charging stations, and these papers do not model recharging decisions at nodes. Consequently, they also do not capture overcharging costs considered in the presented work. 
All of the aforementioned models assume that there is no downtime for refueling or recharging stations, and that a vehicle never needs to wait to use a station. Although this may be valid for gasoline stations, which can typically accommodate multiple vehicles at the same time and often have negligible wait times, it is not necessarily the case for electric charging stations. Most charging stations have only one or two plugs, and due to the long time that it takes to recharge a vehicle, the availability of a charging station can become an issue when multiple vehicles are present. To the best of the authors' knowledge, there is no other work in the literature that addresses this problem of finding an optimal recharging policy when recharging station availability is stochastic. However, there are several papers that consider routing problems with stochastic elements, and we refer the reader to [6] for a review of the existing literature. The body of work that is perhaps most relevant to the study presented in this paper involves anticipatory routing, where the goal is to anticipate information before it becomes available and minimize the total expected travel cost. In [28], a vehicle travels from an origin to a destination, and along the way customers may request service according to known distributions. Customer requests may be realized at any point along the vehicle's path, and the vehicle must respond to all requests. An optimal route therefore is not the shortest path between the origin and destination, but instead includes detours to approach potential customers in the event that they request service. A similar model in which congestion along arcs is the anticipated information is studied in [29], and in both papers, structural properties of optimal anticipatory policies are derived. However, the models only consider routing decisions and not refueling decisions.

\section{Problem setting and motivation}

In this section, we detail the setting of our adaptive routing and recharging problem for electric vehicles. We also provide a motivating example to demonstrate the potential benefits of adaptive routing and recharging.

\subsection{Problem setting}

Consider an EV with zero initial charge that must travel within a network $\mathcal{G}=(\mathcal{N}, \mathcal{E})$ from origin node $o$ to destination node $n$, where $o$ and $n$ are in the node set $\mathcal{N}$, and $\mathcal{E}$ is the set of edges. Every node $i \in \mathcal{N}$ has a charging station, but the availability of each station is uncertain (e.g., due to being occupied by another vehicle) and is denoted by a random variable $A_{i}$. When the vehicle arrives at node $i$ and finds

the station available (i.e., $A_{i}=1$ ), it may begin recharging immediately. In the event that the charging station is unavailable (i.e., $A_{i}=0$ ), the driver must wait a time interval $W_{i}$ until the station becomes 
available before recharging, where $W_{i}$ is also a random variable. We assume that for each $i \in \mathcal{N}$, the driver knows the probability of station $i$ being available, $P\left(A_{i}=1\right)$, and the expected waiting time when it is unavailable, $E\left[W_{i} \mid A_{i}=0\right]$, a priori. Since there is no waiting time when the station is available (i.e., $\left.P\left(W_{i}=0 \mid A_{i}=1\right)=1\right)$, we let $\mathcal{W}_{i}=E\left[W_{i} \mid A_{i}=0\right]$ to simplify the notation. The values of the random variables $A_{i}$ and $W_{i}$ are realized when the $\mathrm{EV}$ arrives at node $i$ and observes the state of the charging station, and they are assumed to be independent of previous realizations observed by the vehicle if it visited the node previously.

As the driver selects a path to travel from $o$ to $n$, he or she must decide which nodes to visit, whether or not to stop and recharge at each visited node (station), and how much to recharge at each stop. The vehicle's charge level can never exceed the maximum capacity of the battery, $q_{\text {max }}$, and it can never drop below zero. We also do not allow the vehicle to discharge energy back to the grid. We let $q_{i}$ denote the charge level of the vehicle when it arrives at node $i$, and for any pair of nodes $i$ and $j$ such that $(i, j) \in \mathcal{E}$, we let $t_{i j}>0$ and $h_{i j}>0$ denote the time and amount of charge, respectively, required to traverse $(i, j)$. We make the following monotonicity assumption for quantities $t_{i j}$ and $h_{i j}$.

Assumption 1 For any pair of edges $(i, j),(k, \ell) \in \mathcal{E}$ we have $t_{i j} \leq t_{k \ell}$ if and only if $h_{i j} \leq h_{k \ell}$.

Then the set of feasible charging amounts at node $i$ prior to traveling along an edge $(i, j)$, which we denote by $R_{i j}\left(q_{i}\right)$, is

$$
R_{i j}\left(q_{i}\right)=\left[\left(h_{i j}-q_{i}\right)^{+}, q_{\max }-q_{i}\right]
$$

We assume that $h_{i j} \leq q_{\max }$ for all $(i, j) \in \mathcal{E}$ to ensure feasibility.

Each time the vehicle stops to recharge, it incurs a fixed stopping cost s. The vehicle also incurs a recharging cost at a rate of $\gamma \geq 0$ per unit of energy recharged plus an additional overcharging cost when its charge level exceeds $\alpha q_{\max }$, where $0<\alpha<1$. This threshold $\alpha q_{\max }$ represents the point at which the charging voltage reaches its maximum value and the charging current begins to decrease. Thus, the overcharging cost takes into account the additional time per unit of energy recharged (caused by the decreasing current). We denote the overcharging cost as $f(z)$, where $z \in\left[0,(1-\alpha) q_{\max }\right]$ is the amount by which the vehicle's charge level exceeds $\alpha q_{\max }$ after recharging, and $f(\cdot)$ is a convex and increasing function with $f(0)=0$. Such properties are reflective of actual battery dynamics that make it undesirable to regularly overcharge the battery. If the vehicle's charge level $(q)$ already exceeds $\alpha q_{\max }$ when it stops to recharge, we discount the overcharging cost by $f\left(q-\alpha q_{\max }\right)$. Therefore, if we let $c(r, q)$ denote the cost of recharging amount of 
energy $r$ when the vehicle's initial charge level is $q$, then we have

$$
c(r, q)=\gamma r+\left[f\left(\left(q+r-\alpha q_{\max }\right)^{+}\right)-f\left(\left(q-\alpha q_{\max }\right)^{+}\right)\right]
$$

See Figure 1 for an example of $c(r, 0)$. We assume that all charging stations have identical cost function $c(\cdot, \cdot)$, which is a reasonable assumption since most public charging stations in existence today have similar hardware configurations (most recharge at 220 volts, with the exception of a few "fast charging" stations that recharge at 440 volts [5]). Furthermore, regional variations in electricity rates are minimal.

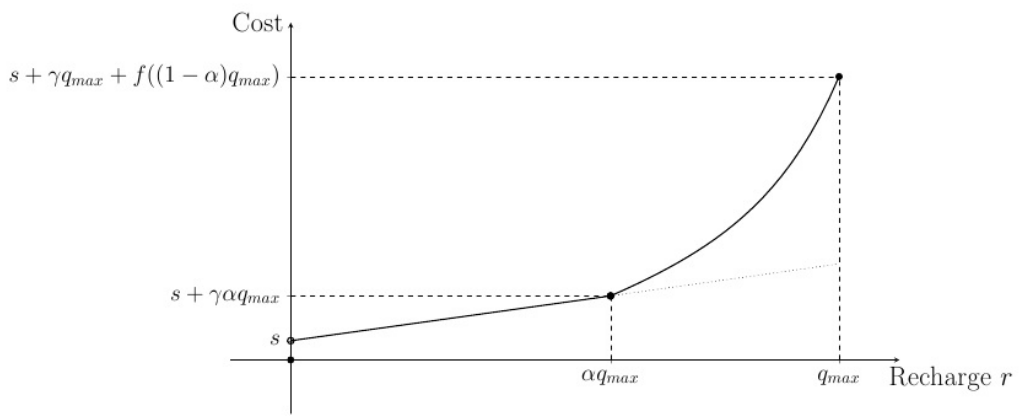

Figure 1: Sample illustration of $c(r, q)$ for $q=0[27]$

We formulate our problem using a dynamic programming approach as follows.

Objective: Find a minimum-cost routing and recharging policy from an origin node $o$ to a destination node $n$. The total cost is measured in units of time and is equal to the sum of all travel time, waiting time for available charging stations, and charging costs (i.e., stopping, recharging and overcharging costs).

State space: The state space, which we denote by $\mathcal{S}$, is defined as

$$
\mathcal{S}=\left\{\left(i, q_{i}, a_{i}\right): i \in \mathcal{N}, q_{i} \in\left[0, q_{\max }\right], a_{i} \in\{0,1\}\right\}
$$

Each state consists of three components: the current vehicle position (node $i$ ), the vehicle's charge level $q_{i}$ upon arrival at the node, and the realized availability of the charging station at the node, denoted by $a_{i}$.

Action space: We denote the action space by

$$
\mathcal{A}_{\left(i, q_{i}\right)}=\left\{\left(j, r_{i}\right):(i, j) \in \mathcal{E}, r_{i} \in R_{i j}\left(q_{i}\right)\right\}
$$

where each action consists of the next node to visit, $j$, along with the amount to recharge at the current node, $r_{i}$. The next node must be adjacent to the current node, and the recharging amount must be feasible 
(where $R_{i j}\left(q_{i}\right)$ is as defined in (1)) to allow the vehicle to reach the next node.

Value function: We let $V\left(i, q_{i}, a_{i}\right)$ denote the value function, which represents the minimum expected cost of traveling to the destination $n$ from node $i$ given that the current state is $\left(i, q_{i}, a_{i}\right)$. We also let $I_{r_{i}>0}$ denote the indicator function that equals 1 if $r_{i}>0$ (i.e., if the vehicle stops to recharge at node $i$ ) and 0 otherwise. Then the value function can be defined recursively as

$$
\begin{aligned}
V\left(i, q_{i}, a_{i}\right)= & \min _{\left(j, r_{i}\right) \in \mathcal{A}_{\left(i, q_{i}\right)}}\left\{t_{i j}+\left(s+E\left[W_{i} \mid A_{i}=a_{i}\right]+c\left(r_{i}, q_{i}\right)\right) I_{r_{i}>0}+E_{A_{j}}\left[V\left(j, q_{i}+r_{i}-h_{i j}, A_{j}\right)\right]\right\} \\
= & \min _{\left(j, r_{i}\right) \in \mathcal{A}_{\left(i, q_{i}\right)}}\left\{t_{i j}+\left(s+\left(1-a_{i}\right) \mathcal{W}_{i}\right) I_{r_{i}>0}+c\left(r_{i}, q_{i}\right)+\right. \\
& {\left.\left[P\left(A_{j}=1\right)\right] V\left(j, q_{i}+r_{i}-h_{i j}, 1\right)+\left[1-P\left(A_{j}=1\right)\right] V\left(j, q_{i}+r_{i}-h_{i j}, 0\right)\right\} }
\end{aligned}
$$

since $E\left[W_{i} \mid A_{i}=1\right]=0$ and $c\left(0, q_{i}\right)=0$ for any value of $q_{i}$. We set $V(n, \cdot, \cdot)=0$ and seek to evaluate $E\left[V\left(o, 0, A_{o}\right)\right]$, the minimum expected total cost of traveling from $o$ to $n$ when the vehicle's initial charge level is zero.

\subsection{Motivation for adaptive decision making}

To see the benefits of adaptive routing and recharging decisions, consider the example shown in Figure 2. The road network consists of nine nodes arranged in a square grid, with the origin $(0,0)$ and destination $(2,2)$ at opposite corners, and $q_{\max }=2 h$, where $h$ is the amount of charge necessary to traverse any edge. The label next to each node on the figure corresponds to the expected wait time when the EV finds the station unavailable upon arrival (i.e., $\mathcal{W}_{i}$ ), and the availability probabilities for each node are 0.5 (i.e., $\left.P\left(A_{i}=1\right)=0.5\right)$. Furthermore, the nodes without any labels (those shaded in light grey) do not have any recharging facilities, that is, $P\left(A_{i}=1\right)=0$ and $\mathcal{W}_{i}=\infty$. For illustration purposes, we consider only waiting costs and neglect any stopping, recharging, overcharging, and traveling costs.

In this setting, an optimal a priori policy, shown in Figure $3(\mathrm{a})$, is to recharge $2 h$ at the origin and $2 h$ at node $(0,2)$ with a total expected waiting cost of $0.5 \cdot(0)+0.5 \cdot(2)=1$. Now consider an adaptive policy where we allow the driver to change the recharging decisions dynamically, but we fix the path to an a priori optimal path, as shown in Figure 3(b). Thus, we allow the vehicle to recharge at any node along the path in response to the observed station availability upon arrival at the node. The optimal policy in such a case is to recharge $2 h$ at the origin, and if the charging station at node $(0,1)$ is unavailable when the vehicle arrives, then the vehicle should recharge 0 at $(0,1), 2 h$ at $(0,2)$, and 0 at $(1,2)$. Otherwise, if the driver finds the 
station at node $(0,1)$ to be available, then the vehicle should recharge $h$ at node $(0,1)$. The EV then does not have to recharge at node $(0,2)$ and will not do so unless that station is also available upon arrival. That is, the driver will recharge $h$ at node $(0,2)$ if the station there is available (and then 0 at $(1,2))$ or will recharge $h$ at $(1,2)$ and 0 at $(0,2)$ if the station at node $(0,2)$ is unavailable. The cost of such a policy is the same as that of the optimal a priori policy when the station at node $(0,1)$ is unavailable, yet when that station is available, then the only scenario in which the expected waiting cost is nonzero is if both the stations at $(0,2)$ and $(1,2)$ are unavailable, in which case the expected waiting cost is 2 . The overall expected waiting cost of the optimal policy with adaptive recharging decisions is therefore $0.5 \cdot(1)+0.5 \cdot(0.75 \cdot(0)+0.25 \cdot(2))=0.75$, which is 0.25 less than the cost of the optimal a priori policy.

In the model where both adaptive routing and recharging decisions are allowed, note that the optimal policy when the station at node $(0,1)$ is available is to recharge $h$ there and then proceed to node $(2,1)$, recharging 0 at $(1,1)$ and $h$ at $(2,1)$ at zero total cost in order to reach the destination (see Figure 3(c)). Otherwise, when the station at node $(0,1)$ is unavailable, the optimal policy is the same as the optimal a priori policy. The cost of the optimal policy with adaptive routing and recharging is thus $0.5 \cdot(0)+0.5 \cdot(1)=0.5$, which is 0.25 less than the cost of the optimal policy with adaptive recharging only and 0.5 less than the cost of the optimal a priori policy. Therefore, both adaptive routing and recharging decisions can lead to a significant reduction in waiting cost.

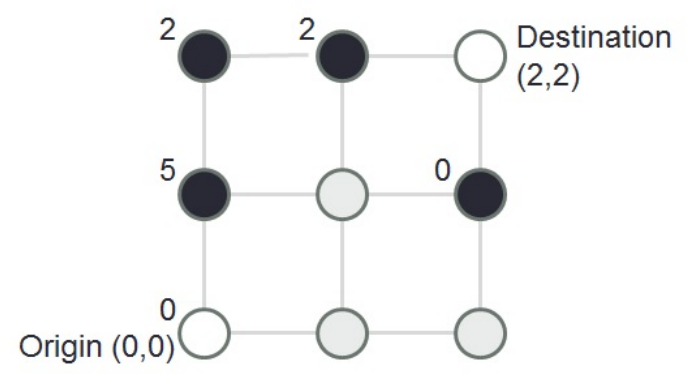

Figure 2: Example network with origin at $(0,0)$ and destination at $(2,2)$. Labels represent $\mathcal{W}_{i}$, and $P\left(A_{i}=\right.$ $1)=0.5$ for all black nodes and the origin (gray nodes do not have charging stations, i.e., $P\left(A_{i}=1\right)=0$ and $\left.\mathcal{W}_{i}=\infty\right)$.

\section{Adaptive recharging policy for a fixed path}

In this section, we solve for an optimal recharging policy along a fixed path $\mathcal{N}=(0, \ldots, n)$ consisting of a sequence of $n+1$ nodes. To simplify the notation, we let $h_{i}$ and $t_{i}$ denote the energy and time, respectively, 


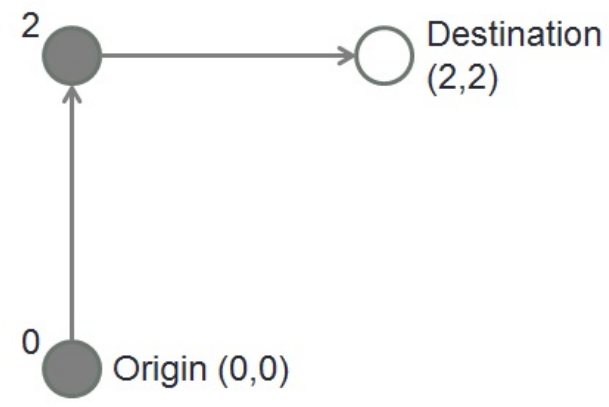

(a) Optimal a priori policy

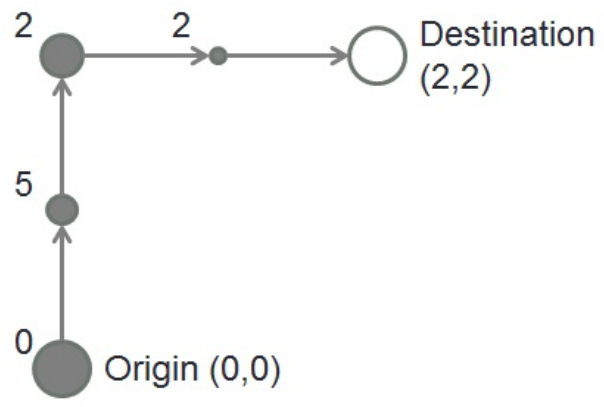

(b) Optimal policy with adaptive recharging

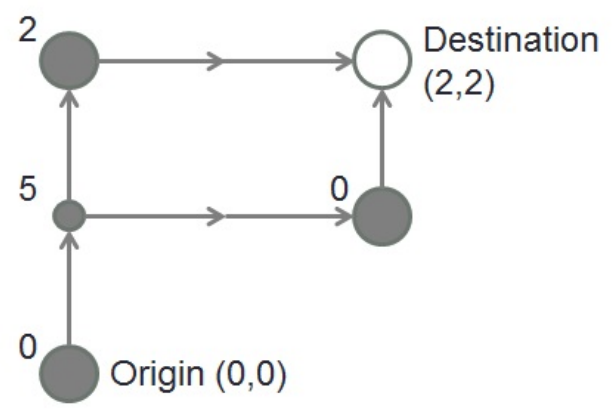

(c) Optimal policy with adaptive routing and recharging

Figure 3: Optimal policies with (a) no adaptivity, (b) adaptive recharging only, and (c) adaptive routing and recharging. The size of each node corresponds to the probability that the vehicle recharges at the node under the specified policy given that the vehicle visits the node, with larger sizes representing higher probabilities.

required to travel from node $i$ to node $i+1$. Due to the fact that there are no routing decisions to be made and the vehicle always travels forward along the path, the choice of which node to visit next is fixed and thus the action space reduces to

$$
\mathcal{A}_{\left(i, q_{i}\right)}=\left\{\left(j, r_{i}\right): j=i+1, r_{i} \in\left[\left(h_{i}-q_{i}\right)^{+}, q_{\max }-q_{i}\right]\right\} .
$$

If we instead embed the fixed path in the value function representation from (3) and use

$$
\mathcal{A}_{\left(i, q_{i}\right)}^{R}=\left\{r_{i}: r_{i} \in\left[\left(h_{i}-q_{i}\right)^{+}, q_{\max }-q_{i}\right]\right\}
$$

to denote the part of the action space consisting only of the feasible recharging amounts at each node, then we can rewrite the value function as

$$
V\left(i, q_{i}, a_{i}\right)=\min _{r_{i} \in \mathcal{A}_{\left(i, q_{i}\right)}^{R}}\left\{t_{i}+\left(s+\left(1-a_{i}\right) \mathcal{W}_{i}\right) I_{r_{i}>0}+c\left(r_{i}, q_{i}\right)+\right.
$$




$$
\left.\left[P\left(A_{i+1}=1\right)\right] V\left(i+1, q_{i}+r_{i}-h_{i}, 1\right)+\left[1-P\left(A_{i+1}=1\right)\right] V\left(i+1, q_{i}+r_{i}-h_{i}, 0\right)\right\} .
$$

To denote a feasible recharging policy for the path, we use $\pi_{\mathcal{N}}^{R}: \mathcal{S} \rightarrow \mathcal{A}^{R}$, with $\pi_{\mathcal{N}}^{R}\left(i, q_{i}, a_{i}\right) \in \mathcal{A}_{\left(i, q_{i}\right)}^{R}$ representing the recharging amount specified by the policy $\pi_{\mathcal{N}}^{R}$ for the state $\left(i, q_{i}, a_{i}\right)$. The expected cost of reaching the end of the path under such a policy for a given state $\left(i, q_{i}, a_{i}\right)$, which we denote $C_{\pi_{\mathcal{N}}^{R}}\left(i, q_{i}, a_{i}\right)$, satisfies

$$
\begin{array}{r}
C_{\pi_{\mathcal{N}}^{R}}\left(i, q_{i}, a_{i}\right)=t_{i}+\left(s+\left(1-a_{i}\right) \mathcal{W}_{i}\right) I_{\pi_{\mathcal{N}}^{R}\left(i, q_{i}, a_{i}\right)>0}+c\left(\pi_{\mathcal{N}}^{R}\left(i, q_{i}, a_{i}\right), q_{i}\right)+ \\
{\left[P\left(A_{i+1}=1\right)\right] C_{\pi_{\mathcal{N}}^{R}}\left(i+1, q_{i}+\pi_{\mathcal{N}}^{R}\left(i, q_{i}, a_{i}\right)-h_{i}, 1\right)+} \\
{\left[1-P\left(A_{i+1}=1\right)\right] C_{\pi_{\mathcal{N}}^{R}}\left(i+1, q_{i}+\pi_{\mathcal{N}}^{R}\left(i, q_{i}, a_{i}\right)-h_{i}, 0\right),}
\end{array}
$$

where $I_{\pi_{\mathcal{N}}^{R}\left(i, q_{i}, a_{i}\right)>0}$ equals 1 if $\pi_{\mathcal{N}}^{R}\left(i, q_{i}, a_{i}\right)>0$ and 0 otherwise. We let $\Pi_{\mathcal{N}}^{R}$ denote the set of all feasible recharging policies for the path $\mathcal{N}$, and thus the value function can be expressed in terms of the cost function $C_{\pi_{\mathcal{N}}^{R}}(\cdot)$ as

$$
V\left(i, q_{i}, a_{i}\right)=\min _{\pi_{\mathcal{N}}^{R} \in \Pi_{\mathcal{N}}^{R}} C_{\pi_{\mathcal{N}}^{R}}\left(i, q_{i}, a_{i}\right)
$$

Finding an optimal recharging policy is not practical when the vehicle's charge level can take on any value in the interval $\left[0, q_{\max }\right]$ since this corresponds to a continuous action space and state space. However, without loss of optimality, we can restrict the possible values for the recharging amounts at each node to a discrete set. We then consider only finitely many values for the vehicle's charge level, thereby making the problem of finding an optimal recharging policy tractable. The following two lemmas show that there exists an optimal recharging policy such that all of the recharging amounts (and also incoming charge levels at each node) can be expressed as sums of the $h_{i}$ values.

Lemma 1 There exists an optimal recharging policy $\pi_{\mathcal{N}}^{{ }^{*}}: \mathcal{S} \rightarrow \mathcal{A}^{R}$ such that

$$
\pi_{\mathcal{N}}^{R^{*}}\left(i, q_{i}, a_{i}\right) \in\left\{\left(\sum_{\ell=i}^{k-1} h_{\ell}-q_{i}\right)^{+}: k \in(i+1, \ldots, n-1), \sum_{\ell=i}^{k-1} h_{\ell} \leq q_{\max }\right\}
$$

for all $\left(i, q_{i}, a_{i}\right) \in \mathcal{S}$.

Proof. See Appendix A.2.1

Since we assume that the vehicle's charge level is zero at the beginning of the path, it follows from Lemma 1 that there exists an optimal recharging policy such that every incoming charge level and recharging amount 
can be expressed as a summation of the $h_{\ell}$ parameters. We prove this claim in the following Lemma 2.

Lemma 2 Let

$$
\mathcal{H}(i)=\left\{\sum_{\ell=i}^{k-1} h_{\ell}: k \in(i, \ldots, n-1), \sum_{\ell=i}^{k-1} h_{\ell} \leq q_{\max }\right\}
$$

denote the set of feasible charge levels at node $i$ that can be expressed as a summation of the $h_{\ell}$ parameters. Then there exists an optimal recharging policy $\pi_{\mathcal{N}}^{R *}: \mathcal{S} \rightarrow \mathcal{A}^{R}$ such that $q_{i}=\sum_{\ell=i}^{k-1} h_{\ell} \in \mathcal{H}(i)$ for some $k \in(i, \ldots, n-1)$ and $\pi_{\mathcal{N}}^{R}\left(i, q_{i}, a_{i}\right) \in \mathcal{H}(k)$ for any realized state $\left(i, q_{i}, a_{i}\right) \in \mathcal{S}$, given that the initial state is $(0,0, \cdot)$

Proof. See Appendix A.2.2

By Lemma 2, without loss of optimality, we can create a value function representation that considers only a discrete set of values for both the charge level and recharging amount at each node. If we let $V\left(i, k, a_{i}\right)$ denote the value function representing the minimum expected cost of reaching node $n$ when we arrive at node $i$ with charge level $\sum_{\ell=i}^{k-1} h_{\ell}$ (i.e., just enough to reach node $k$ ) and the station availability is $a_{i}$, then we can define the value function recursively as

$$
\begin{array}{r}
V\left(i, k, a_{i}\right)=\min _{j \in(k, \ldots, n): \sum_{\ell=i}^{j-1} h_{\ell} \leq q_{\max }}\left\{t_{i}+\left(s+\left(1-a_{i}\right) \mathcal{W}_{i}\right) I_{j>k}+c\left(\sum_{\ell=k}^{j-1} h_{\ell}, \sum_{\ell=i}^{k-1} h_{\ell}\right)+\right. \\
\left.\left[P\left(A_{i+1}=1\right)\right] V(i+1, j, 1)+\left[1-P\left(A_{i+1}=1\right)\right] V(i+1, j, 0)\right\},
\end{array}
$$

where $I_{j>k}$ equals 1 if $j>k$ (i.e., if the vehicle stops to recharge) and 0 otherwise, and $\sum_{\ell=k}^{j-1} h_{\ell}$ is the recharging amount. Then, solving the recursive equation $(4)$ until we find $V(0,0, \cdot)$ will result in an optimal recharging policy along the given path.

Proposition 3 The computational time required to find an optimal policy that solves recursive equation (4) is $O\left(n^{3}\right)$.

Proof. For each pair of nodes $i, k \in \mathcal{N}$ and charging station availability $a_{i} \in\{0,1\}$, equation (4) chooses a node $j$ from a subset of $\mathcal{N}$. Since $|\mathcal{N}|=n$, the recursive equation can be solved using Dijkstra's algorithm [3] in $O\left(n^{3}\right)$. 


\section{$5 \quad$ Adaptive routing and recharging for a general network}

Although solving the dynamic programming formulation in (4) to obtain an optimal recharging policy along a fixed path is straightforward, applying a similar solution approach to find an optimal policy within a network is more difficult due to the need to incorporate routing decisions as well. The decision space increases to include the next node to visit on the EV route and the set of states to be considered increases to include potentially all nodes of the road network. Furthermore, since the order of the visited nodes is not predetermined as it is in the fixed path setting, value function updates could require re-evaluation of previously explored paths and policies. At the same time, the routing decisions between recharging stops are integrated into the adaptive decision making since they impact the nodes that the EV visits as potential recharging locations if they happen to be available. These facts lead to significant computational inefficiencies unless the number of calculations can be reduced. In Section 5.1, we present an in-depth analysis of the computational complexity of adaptive routing and recharging in a general network, which motivates our solution approach. Rather than attempt to solve for an optimal policy directly, we develop heuristic approaches that build on our analysis and findings for a priori policies. Section 5.2 presents these optimal a priori policies, and Section 5.3 describes the heuristics for adaptive policies.

\subsection{Problem complexity analysis}

To find an adaptive routing and recharging policy for a general network, we return to functional equation (3). The first obstacle to recursively solving (3) that we must address is the infinite set of actions $\mathcal{A}_{\left(i, q_{i}\right)}$ (and as a result, the infinite set of states $\mathcal{S}$ ) due to the continuous set of values that the recharging amount $r_{i}$ and charge level $q_{i}$ can take. To overcome this challenge, we extend the results of Lemma 2 to the general network routing and recharging problem. Before we do so, however, we introduce some additional notation and definitions.

For any two nodes $i, j \in \mathcal{N}$, let $\mathcal{P}_{q}(i, j)$ denote the set of paths from $i$ to $j$ with total required charge level of less than or equal to $q$ (where $q \leq q_{\max }$ ). As will be discussed later in further detail, we allow paths in $\mathcal{P}_{q}(i, j)$ to contain cycles. Then, let $\mathcal{Q}_{q}(i, j)$ denote the set of charge levels required by paths in $\mathcal{P}_{q}(i, j)$ (by construction, the quantities in this set will be bounded by zero and $q)$. We let $\mathcal{P}_{q}(i, j)=\emptyset$ and $\mathcal{Q}_{q}(i, j)=\emptyset$ if no such paths exist, and we define

$$
\mathcal{Q}_{q}(i):=\bigcup_{j \in \mathcal{N}} \mathcal{Q}_{q}(i, j)
$$

Finally, as before, we denote a feasible routing and recharging policy by $\pi_{\mathcal{N}}: \mathcal{S} \rightarrow \mathcal{A}$, with $\pi_{\mathcal{N}}\left(i, q_{i}, a_{i}\right) \in$ 
$\mathcal{A}_{\left(i, q_{i}\right)}$ representing the next node to visit and the recharging amount specified by the policy $\pi_{\mathcal{N}}$ for the state $\left(i, q_{i}, a_{i}\right)$. We are now ready to state the lemma.

Lemma 4 There exists an optimal policy $\pi_{\mathcal{N}}^{*}: \mathcal{S} \rightarrow \mathcal{A}$ for the recursive equation (3) such that

$$
\pi_{\mathcal{N}}^{*}\left(i, q_{i}, a_{i}\right) \in\left\{\left(j, r_{i}\right):(i, j) \in \mathcal{E}, r_{i}+q_{i}-h_{i j} \in \mathcal{Q}_{\left(q_{\max }-h_{i j}\right)}(j)\right\}
$$

for all $\left(i, q_{i}, a_{i}\right) \in \mathcal{S}$.

In words, Lemma 4 states that there exists a policy such that we would only recharge an amount corresponding to the exact charge required to reach some node in $\mathcal{N}$ from the current node. The proof for Lemma 4 is similar to the proof for Lemma 1 and is therefore omitted.

Since we assume the initial charge level to be zero, we can also restrict the set of charge levels considered in our state space $\mathcal{S}$ to a discrete set, without loss of optimality. We do so in the following corollary.

Corollary 5 There exists an optimal policy $\pi_{\mathcal{N}}^{*}: \mathcal{S} \rightarrow \mathcal{A}$ to the recursive equation $(3)$ such that $q_{i} \in \mathcal{Q}_{q_{\max }}(i)$ for any realized state $\left(i, q_{i}, a_{i}\right) \in \mathcal{S}$, given that the initial state is $(0,0,$.$) .$

While Lemma 4 and Corollary 5 deliver finite action and state spaces, the sizes of those sets remain large due to the fact that an optimal policy might include cycles. The following propositions establish the cycling property of the adaptive routing and recharging policies for a general network and the functional equation (3).

Proposition 6 An optimal policy satisfying the properties established in Lemma 4 and Corollary 5 may visit a node in $\mathcal{N}$ more than once. That is, a vehicle might cycle on the network.

Proof. Consider an undirected network $\mathcal{G}=(\mathcal{N}, \mathcal{E})$, where $\mathcal{N}=\{0,1,2,3\}, \mathcal{E}=\{(0,1),(1,2),(1,3)\}$, node 0 is the origin, and node 3 is the destination (see Figure 4). Let $q_{\max } \leq h_{01}+h_{13}, h_{01}+h_{12} \leq q_{\max }$, and $h_{12}+h_{13} \leq q_{\max }$. That is, having a full charge level is not sufficient to get from origin to destination without recharging along the way, and the vehicle must recharge either at node 1 or 2 before proceeding to node 3 . Furthermore, we let $\mathcal{W}_{1}>0$ and $\mathcal{W}_{2}=0$. Then consider the following two policies, $\pi^{1}$ and $\pi^{2}$.

- Policy $\pi^{1}$ : Recharge $r_{0}=h_{01}$ at node 0 and travel to node 1 . Recharge $r_{1}=h_{13}$ at node 1 (with expected waiting time $\mathcal{W}_{1}$ if $\left.A_{1}=0\right)$ and travel directly to node 3 .

- Policy $\pi^{2}$ : Recharge $r_{0}=h_{01}+h_{12}$ at node 0 and travel to node 1 . If $A_{1}=1$ (i.e., the recharging station at node 1 is available), then recharge $r_{1}=h_{13}-h_{12}$ at node 1 and travel directly to node 3 . Otherwise, 
if $A_{1}=0$, then do not recharge at node 1, but instead travel to node 2, recharge $r_{2}=h_{12}+h_{13}$ there, and complete the trip by traveling to node 1 and then to node 3 .

Note that $\pi^{1}$ is the minimum cost policy among all the feasible policies that do not include a cycle on network $\mathcal{G}$, and policy $\pi^{2}$ contains a cycle.

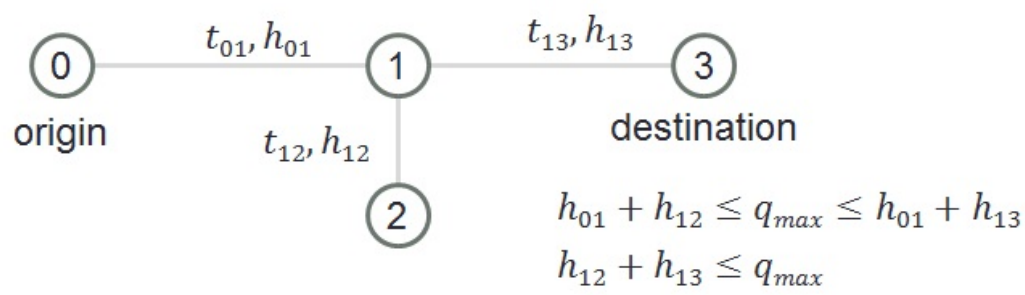

Figure 4: Illustrative example of network $\mathcal{G}=(\mathcal{N}, \mathcal{E})$ for the proof of Proposition 6.

Then the costs of policies $\pi^{1}$ and $\pi^{2}$, respectively, are

$$
\begin{array}{cc}
C_{\pi^{1}}= & s+c\left(h_{01}, 0\right)+t_{01}+\left[1-P\left(A_{1}=1\right)\right] \mathcal{W}_{1}+s+c\left(h_{13}, 0\right)+t_{13}, \\
C_{\pi^{2}}= & s+c\left(h_{01}+h_{12}, 0\right)+t_{01}+P\left(A_{1}=1\right)\left[s+c\left(h_{13}-h_{12}, h_{12}\right)\right]+ \\
& {\left[1-P\left(A_{1}=1\right)\right]\left[2 t_{12}+s+c\left(h_{12}+h_{13}, 0\right)\right]+t_{13}} \\
= & 2 s+\left[c\left(h_{01}, 0\right)+c\left(h_{12}, h_{01}\right)\right]+t_{01}+P\left(A_{1}=1\right)\left[c\left(h_{13}, 0\right)-c\left(h_{12}, 0\right)\right]+ \\
& {\left[1-P\left(A_{1}=1\right)\right]\left[2 t_{12}+c\left(h_{13}, 0\right)+c\left(h_{12}, h_{13}\right)\right]+t_{13} .}
\end{array}
$$

Then,

$$
\begin{aligned}
C_{\pi^{1}}-C_{\pi^{2}} & =\left[1-P\left(A_{1}=1\right)\right]\left[\mathcal{W}_{1}-2 t_{12}-c\left(h_{12}, h_{13}\right)\right]-c\left(h_{12}, h_{01}\right)+P\left(A_{1}=1\right) c\left(h_{12}, 0\right) \\
& =\left[1-P\left(A_{1}=1\right)\right]\left[\mathcal{W}_{1}-2 t_{12}-c\left(h_{12}, h_{13}\right)-c\left(h_{12}, 0\right)\right]-c\left(h_{12}, h_{01}\right)+c\left(h_{12}, 0\right) \\
& \geq\left[1-P\left(A_{1}=1\right)\right] \mathcal{W}_{1}-2 t_{12}-c\left(h_{12}, h_{13}\right)-c\left(h_{12}, h_{01}\right) \quad\left(\text { since } 0 \leq P\left(A_{1}=1\right)<1\right) \\
& >0 \text { if }\left[1-P\left(A_{1}=1\right)\right] \mathcal{W}_{1}>2 t_{12}+c\left(h_{12}, h_{13}\right)+c\left(h_{12}, h_{01}\right) .
\end{aligned}
$$

That is, it is advantageous to cycle between nodes 1 and 2 if the expected waiting time at node 1 is greater than the extra travel and charge time required for the detour. Since policy $\pi^{1}$ is the minimum-cost cycle-free feasible policy, this proves the required statement.

Note that the example presented in Figure 4 can be modified so that a vehicle might repeat the same cycle multiple times. Let $P\left(A_{1}=1\right)=0$ (i.e., there is no charging station at node 1 ) and let $\mathcal{W}_{2}>0$. 
Suppose that the vehicle arrives at node 2 with charge level $q_{2}$ and finds the charging station unavailable. It can either wait with expected cost $\mathcal{W}_{2}$ or, if its charge level is at least $2 h_{12}$, it can travel to node 1 and return to node 2 in order to observe a new realization of $A_{2}$. The vehicle therefore would prefer to cycle between nodes 1 and 2 if

$$
\begin{aligned}
\mathcal{W}_{2}+c\left(h_{12}+h_{13}-q_{2}, q_{2}\right) & >2 t_{12}+\left[1-P\left(A_{2}=1\right)\right] \mathcal{W}_{2}+c\left(h_{12}+h_{13}+2 h_{12}-q_{2}, q_{2}-2 h_{12}\right) \\
& =2 t_{12}+\left[1-P\left(A_{2}=1\right)\right] \mathcal{W}_{2}+c\left(h_{12}+h_{13}-q_{2}, q_{2}\right)+c\left(2 h_{12}, q_{2}-2 h_{12}\right) \\
\Rightarrow \quad P\left(A_{2}=1\right) \mathcal{W}_{2} & >2 t_{12}+c\left(2 h_{12}, q_{2}-2 h_{12}\right) .
\end{aligned}
$$

Intuitively, if $P\left(A_{2}=1\right)$ and $\mathcal{W}_{2}$ are relatively large quantities (i.e., the station at node 2 is likely to be available, but if it is not, then it remains unavailable for a while) and the driver finds that the station at node 2 is unavailable, then the cost of revisiting node 1 and returning to node 2 in order to observe a different realization of the station's availability is favorable to the driver. The driver may even cycle multiple times between the two nodes until a favorable observation occurs. In a realistic setting, however, this phenomenon is unlikely to occur since the availability of a given charging station is not truly memoryless or independent of previous observations as it is in our model. One would not expect a station to become available sooner by leaving it for a while and returning later as opposed to staying and waiting for it to become available. Nevertheless, we formalize the repeated cycle property in the following proposition.

Proposition 7 An optimal policy might traverse a given cycle in network $\mathcal{G}$ more than once.

We are now ready to conclude the computational complexity analysis of solving recursive equation (3). In order to build a discrete action set $\mathcal{A}_{\left(i, q_{i}\right)}$, we first construct a set of paths $\mathcal{P}_{q_{\max }}(i, j)$ for all $j \in \mathcal{N}$. As a result of Proposition 7 , we know that paths in $\mathcal{P}_{q_{\max }}(.,$.$) might contain numerous and repeating cycles.$ Therefore, the same nodes from set $\mathcal{N}$ may appear on a single path multiple times and the only possible bound on the total number of nodes visited by a path is $q_{\max } / \min _{(i, j) \in \mathcal{E}}\left(h_{i j}\right)$, which in the extreme case might approach infinity as $\min _{(i, j) \in \mathcal{E}}\left(h_{i j}\right) \rightarrow 0$. Intuitively, as the arc lengths become shorter, the number of possible paths that may be traversed with a charge level of $q_{\max }$ increases and, in the limit, approaches infinity. Furthermore, even if we build a discrete action set, solving the dynamic program that allows cycles and has a large action space is prohibitively expensive and no polynomial computational bound can be derived.

This analysis motivates our heuristic solution approaches to an adaptive routing and recharging problem for a general network, discussed in Section 5.3. We propose two heuristic methods that aim to restrict the 
set of paths considered by the adaptive policy to improve the problem's tractability. We use an a priori policy to guide the decision of what the restricted set of paths should be. The a priori policies are easy to implement, they identify promising charging locations for an adaptive policy to visit, and they allow us to benchmark our adaptive approach to assess the value of adaptivity. The first heuristic method uses an a priori policy to select a path to travel. Then, a vehicle commits to the path but can dynamically adapt recharging locations along this path. In the second solution approach, the EV is allowed to adapt its path and recharging locations in response to realized station availabilities while using an a priori policy to restrict the set of paths considered. Next, we discuss the a priori policies (Section 5.2) before detailing the heuristics that build on them (Section 5.3).

\subsection{Optimal a priori policies}

In this section, we identify optimal a priori policies where both the vehicle's route and recharging locations are selected before departing the origin and do not change en route based on the realized availability of charging stations. Not only is an optimal a priori policy easier to obtain, but it is also a feasible policy when adaptive decision making is permitted. As a result, the value of such a policy serves as an upper bound on the value of an optimal adaptive policy. We apply this fact to our later analysis of the adaptive decision making and use the optimal a priori policy as a starting point for the solution procedure to find an optimal adaptive policy. In this section we present algorithms for finding an optimal a priori policy along a path (Section 5.2.1) and within a general network (Section 5.2.2).

\subsubsection{Optimal a priori policy for a fixed path}

Here we examine how to determine an optimal a priori recharging policy along a fixed path $\mathcal{N}=(0, \ldots, n)$ consisting of a sequence of $n+1$ nodes. As in Section 4, we let $h_{i}$ and $t_{i}$ denote the energy and time, respectively, required to travel from node $i$ to node $i+1$. We let $\pi_{\mathcal{N}}^{0}=\left(r_{0}, \ldots, r_{n-1}\right)$ denote an a priori policy, which consists of the sequence of amounts to recharge at each node along the path $\mathcal{N}$ (except node $n$, which is the end of the path). Notice that the amounts to recharge at each node are not dependent on the realized availability of charging stations. In such a policy, the expected waiting time at a node if the vehicle recharges there is

$$
E\left[W_{i}\right]=\left[P\left(A_{i}=1\right)\right] E\left[W_{i} \mid A_{i}=1\right]+\left[1-P\left(A_{i}=1\right)\right] E\left[W_{i} \mid A_{i}=0\right]=\left[1-P\left(A_{i}=1\right)\right] \mathcal{W}_{i}
$$


for all $i \in(1, \ldots, n)$, where $E\left[W_{i} \mid A_{i}=1\right]=0$ since the vehicle does not need to wait when the charging station is available. We show in the following lemma that there exists an optimal a priori policy in which the vehicle only stops to recharge when its charge level is zero. (It is worth noting that while these results are analogous to the zero-inventory-ordering policies in inventory control literature, they do not directly follow from that literature since such policies are not even optimal in the presence of replenishment constraints [7].)

Lemma 8 There exists an optimal a priori recharging policy such that for all $i \in(0, \ldots, n-1), r_{i}>0$ if and only if $q_{i}=0$.

Proof. Let $\pi_{\mathcal{N}}^{0}=\left(r_{0}, \ldots, r_{n-1}\right)$ be an a priori recharging policy. Suppose the vehicle recharges at node $j$ in the policy, and $k$ is the next node along the path where the vehicle recharges (i.e., $\left.k=\min \left\{\ell: \ell>j, r_{\ell}>0\right\}\right)$. Let $\Delta=r_{j}-\sum_{\ell=j}^{k-1} h_{\ell}$ denote the amount recharged at node $j$ that is not used by the time the vehicle reaches node $k$, where $\Delta \geq 0$. Then a policy $\hat{\pi}_{\mathcal{N}}^{0}=\left(\hat{r}_{1}, \ldots, \hat{r}_{n}\right)$ that satisfies

$$
\hat{r}_{i}= \begin{cases}r_{j}-\Delta, & i=j \\ r_{k}+\Delta, & i=k \\ r_{i}, & \text { otherwise }\end{cases}
$$

is also feasible. Furthermore, by the convexity of $c(\cdot)$, the cost of the policy $\hat{\pi}_{\mathcal{N}}^{0}$ is no greater than that of $\pi_{\mathcal{N}}^{0}$. Therefore, there exists an optimal policy in which $r_{i}>0$ implies $q_{i}=0$ for all $i \in(0, \ldots, n-1)$. Conversely, $q_{i}=0$ necessarily implies that $r_{i}>0$ in order to ensure feasibility. It follows that there exists an optimal a priori recharging policy such that for all $i \in(0, \ldots, n-1), r_{i}>0$ if and only if $q_{i}=0$.

If we let $V(i)$ denote the value function representing the minimum expected cost of reaching node $n$ (the end of the path) when the vehicle arrives at node $i$ with zero charge level, then we can define the value function recursively as

$$
V(i)=\min _{j \in(i+1, \ldots, n): \sum_{\ell=i}^{j-1} h_{\ell} \leq q_{\max }}\left\{s+\left[1-P\left(A_{i}=1\right)\right] \mathcal{W}_{i}+c\left(\sum_{\ell=i}^{j-1} h_{\ell}, 0\right)+\sum_{\ell=i}^{j-1} t_{\ell}+V(j)\right\}
$$

where $V(n)=0$. Solving recursive equation (5) for $V(0)$ yields an optimal recharging policy for a given path. This computation can be done using Dijkstra's algorithm [3] in time $O\left(n^{2}\right)$. Note that when the quantity $\left[1-P\left(A_{i}=1\right)\right] \mathcal{W}_{i}$ is the same for all $i \in(0, \ldots, n-1)$, the problem is identical to the one studied in [27] and can be solved more efficiently by removing from the action space values of $j$ for which $\sum_{\ell=i}^{j-1} h_{\ell} \leq \alpha q_{\max }$. 
Nevertheless, one of the contributions of this present work is to capture the heterogeneous charging stations availability when making routing and recharging decisions.

\subsubsection{Optimal a priori policy for a general network}

We return to the general network problem defined in Section 3.1 on a network $\mathcal{G}=(\mathcal{N}, \mathcal{E})$. An optimal a priori policy in a general network must specify not only where to recharge on the way from an origin node $o$ to a destination node $n$, but also the route that the vehicle takes. Since an a priori policy commits to a sequence of recharging locations regardless of whether the vehicle finds the charging stations available or not, the functional equation (3) is modified to remove station availability from the state space, resulting in $(6)$,

$$
V\left(i, q_{i}\right)=\min _{\left(j, r_{i}\right) \in \mathcal{A}_{\left(i, q_{i}\right)}}\left\{t_{i j}+\left(s+\left[1-P\left(A_{i}=1\right)\right] \mathcal{W}_{i}+c\left(r_{i}, q_{i}\right)\right) I_{r_{i}>0}+V\left(j, q_{i}+r_{i}-h_{i j}\right)\right\} .
$$

Despite adding routing decisions to the problem scope, the properties from Section 5.2.1 can be easily extended to the network setting. Since an a priori policy returns a single path (we still must find that path, but a path nevertheless), the property of Lemma 8 can be extended to the network.

Lemma 9 There exists an optimal a priori policy for functional equation (6) such that for all $i \in \mathcal{N}, r_{i}>0$ if and only if $q_{i}=0$.

Proof. The proof follows from Lemma 8 since for any path returned from solving (6), we can apply Lemma 8, considering that path as a fixed path.

From Lemma 9, it follows that we can further simplify the recursive equation (6) by redefining the DP state as the next node $i$ from $\mathcal{N}$ where the EV recharges. Then, the action corresponds to selecting a node $j \in \mathcal{N}$ where the vehicle recharges next. From Property 1 we know that, without loss of optimality, a vehicle should follow the shortest (or minimum required charge) path from $i$ to $j$ and recharge at node $i$ the exact amount needed to traverse the path. We let $t_{p(i, j)}^{*}$ and $h_{p(i, j)}^{*}$ denote the travel time and charge amount, respectively, for such a path. The resulting functional equation one must solve to find an optimal a priori policy on a general network is

$$
V(i)=\min _{j \in \mathcal{N}: h_{p(i, j)}^{*} \leq q_{\max }}\left\{t_{p(i, j)}^{*}+s+\left[1-P\left(A_{i}=1\right)\right] \mathcal{W}_{i}+c\left(h_{p(i, j)}^{*}, 0\right)+V(j)\right\} .
$$

Due to the fact that the a priori policy commits to recharging locations and does not adapt to realized station availabilities, the a priori DP functional equation (7) will only visit each DP state (as well as each 
node in set $\mathcal{N})$ at most once. As a result, the computational complexity of recursively solving (7) is $O\left(n^{4}\right)$ where $n=|\mathcal{N}|$ since for each node in set $\mathcal{N}$, our action space is a subset of $\mathcal{N}$, and we must solve a shortest path problem from the current node to the next recharging node to find $t_{p(i j)}^{*}$ and $h_{p(i j)}^{*}$, which can be done using Dijkstra's algorithm in time $O\left(n^{2}\right)$. Note that while the solution to (7) returns the ordered list of recharging locations, we can also recover the actual paths traversed between those locations at no additional computational cost since we already compute those paths to find the values $t_{p(i j)}^{*}$ and $h_{p(i j)}^{*}$.

\subsection{Heuristic Solution Adaptive Routing and Recharging for a General Network}

As seen in Section 5.2, finding an optimal a priori policy for a general network is computationally tractable and can be done in polynomial time, unlike the case of an adaptive policy. Therefore, we use a priori policies to guide our heuristic solution approaches.

\subsubsection{Heuristic Approach to Adaptive Recharging}

Our first heuristic finds an optimal policy when the vehicle is only allowed to adapt the charging locations dynamically but not the path while en route. The heuristic is broken down into a two-stage solution approach: at the first stage we select which path the EV should follow from origin to destination, and then the second stage selects an adaptive recharging policy for the chosen path. As shown in Section 4, an optimal recharging policy along a fixed path can be obtained using the formulation (4). Thus, the two-step solution approach of our heuristic follows.

Step 1. Solve recursive equation (7) to find an optimal a priori path from origin $o$ to destination $n$.

Step 2. For the path found in Step 1, solve recursive equation (4) to find an optimal recharging policy.

The computational complexity of Heuristic 1 is $O\left(n^{4}\right)$ since Step 1 is performed once in $O\left(n^{4}\right)$, and Step 2 is executed once in $O\left(n^{3}\right)$. The solution found in Step 1 is an upper bound on the adaptive recharging policy since the EV is always able to recharge at the locations identified by the a priori policy. At the same time, if the vehicle encounters an available station before reaching an a priori charging stop, it may be beneficial to recharge there in order to avoid a potential wait in the future. Furthermore, the adaptive policy will also capture the trade-off between recharging sooner than planned with zero waiting cost and potentially longer charging times and additional stops later due to opportunity charging of a non-empty battery. 
One might improve the found solution by considering a larger number of paths in Step 1. Since we still want to ensure that the evaluated paths do not include large detours, finding $k$-shortest paths (e.g., [30]) using an a priori approach and then solving recursive equation (4) for each of them can result in an overall better solution.

\subsubsection{Heuristic Approach to Adaptive Recharging and Routing}

As we saw from an earlier example, it might be beneficial not only to adapt recharging locations along a path, but also to deviate from the path when the vehicle finds an intended recharging location unavailable. While it is intractable to find an optimal adaptive policy to recursive equation (3), we can use a variety of approaches to restrict the subset of paths considered by the policy. Here, we propose a few solution approaches that can be applied to a general network problem. However, without additional structure of the network $\mathcal{G}$, it is not clear which approach is more advantageous, especially when considering the trade-off among implementation complexity, runtime, and performance. In the following Section 6, we present further in-depth analysis and solution approaches to a network with a grid structure, and in Section 7 we conduct a numerical analysis in a realistic setting.

Recursive a priori path Note that for the adaptive recharging policy found using the solution approach described in Section 5.3.1, when an EV stops to recharge at an available station that differers from an optimal a priori location, the optimal a priori policy from that node forward might change. Thus, we can improve the found solution by executing Steps 1 and 2 of the adaptive recharging policy again to allow the vehicle to also adapt its path along the way.

Value function approximation Since an optimal a priori policy found by solving (7) provides an upper bound on the value function for an optimal adaptive policy in recursive equation (3), we can use it to approximate the value function and apply approximate dynamic programming techniques [18] to heuristically solve for an adaptive policy. Note that since the a priori solution provides an upper bound, and not a lower bound, we cannot implement an $A^{*}$ heuristic.

\section{Adaptive policies for a grid network}

In this section, our analysis of the optimal EV routing is carried out on a square grid network where the amount of charge required to traverse each $\operatorname{arc}$ is a constant and denoted by $h$ (i.e., $\left.h_{i j}=h, \forall(i, j) \in \mathcal{E}\right)$. We consider this special case due to several favorable properties of the square grid network that lend themselves 
well to a dynamic programming framework and broad set of applications. Specifically, consider an $x-, y^{-}$ coordinate system imposed on our grid network where, without loss of generality, we assume that the origin $o$ has coordinates $(0,0)$ and the destination $n$ has coordinates $(x, y)(x \geq 0, y \geq 0)$. Then the minimum distance between two nodes can easily be calculated as the sum of the absolute differences between their coordinates. Furthermore, network nodes can be partitioned by the total sum of their $x$ - and $y$ - coordinates such that all nodes in one partition have the same sum values. As a result of such a procedure, all nodes along each $-\pi / 4$-angled diagonal line are grouped together (see Figure 5 ). Indexing the partitions by the values of the sum of the two coordinates of their member nodes creates a natural ordering. In addition, it can be observed that whenever the vehicle travels to an adjacent node, the index of the diagonal passing through its location either increases by one (when the vehicle travels up or to the right) or decreases by one (when it travels down or to the left). By our convention, the vehicle starts its travel on diagonal 0 and ends on diagonal $x+y$. It follows that for any direct path (i.e., shortest or minimum-charge path) between origin and destination in which the vehicle only travels either up or to the right, the distance is simply the difference between the partition indices of the two nodes, or $x+y$. This notion of a direct path (as well as indirect path - a path with total distance greater than the minimum) will be used in our subsequent analysis.

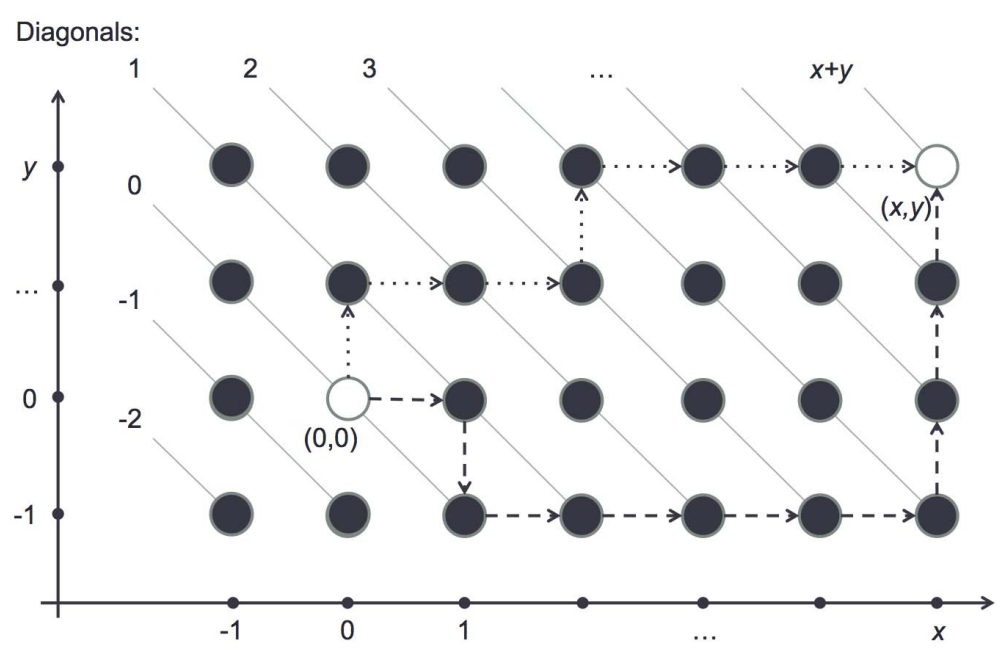

Figure 5: Nodes partitioned diagonally; all nodes along the same diagonal have coordinates that sum to the same value. The dotted line is an example of a direct path from the origin to the destination, and the dashed line is an example of an indirect path.

Besides adding structure to our adaptive routing and recharging problem, a grid network also naturally lends itself to application. Most urban networks (e.g., New York City, Chicago) have grid-like road networks where each node corresponds to an intersection. In more suburban and rural settings where road networks are 
more sparse, a grid still can be used to approximate the road network by setting edge costs, station availability probabilities, and waiting times to arbitrary large values for the absent road links and intersections. A similar approximation scheme can be used to represent the national road network and recharging infrastructure for long-range trips.

Due to computational demands described in Section 5, we develop heuristic solution approaches to estimate the value of adaptive decision making and use an optimal a priori policy to benchmark the improvement of EV performance resulting from introducing adaptivity. The grid network structure allows us to develop a more efficient algorithm to find an optimal a priori policy, which we present in Section 6.1. Then in Section 6.2, we present two heuristics for adaptive policies: the first allows us to compute the benefit of adaptive recharging decisions only, and then we determine the additional savings that result from adaptive routing decisions and recharging strategies.

\subsection{Optimal a priori policy for a grid network}

\subsubsection{Algorithm for optimal a priori policy for a grid network}

When searching for an optimal a priori policy in a network, it is important to establish a tight upper bound quickly on its value. This would allow us to significantly reduce the number of network nodes to be considered as part of an optimal path and prevent us from making unnecessary value function updates at nodes that are located far from both the origin and destination. Our solution approach outlined in Algorithm 1 takes advantage of the grid network structure and quickly computes an upper bound on the value of an optimal a priori policy. It then tightens this bound by searching for policies corresponding to longer paths with potential savings in waiting times at the charging stations. The algorithm terminates once it is established that the current upper bound is also a lower bound and the corresponding policy is optimal.

An upper bound on the total cost of an optimal a priori policy is first calculated by the FindOptDirectPath procedure (detailed in Appendix A.1), which finds an optimal policy over all direct paths between origin and destination. Such calculation can be performed efficiently since the vehicle is restricted to traveling either up or to the right toward the destination node, never detouring outside of the bounding box formed by the origin and destination or moving in a direction away from the destination. In addition, the identified policy corresponds to a shortest path since the length of any direct path is $x+y$. Thus, the FindOptDirectPath procedure finds for each of $x y$ nodes the next node at which to recharge, which is bounded by the distance

from the current node by $k=\left\lfloor\frac{q_{\max }}{h}\right\rfloor$. The nodes considered for the next recharging location therefore are restricted to $O\left(k^{2}\right)$ or $O(x y)$, whichever is smaller. The procedure then runs in $O\left(\min \left(x y k^{2},(x y)^{2}\right)\right)$, and 


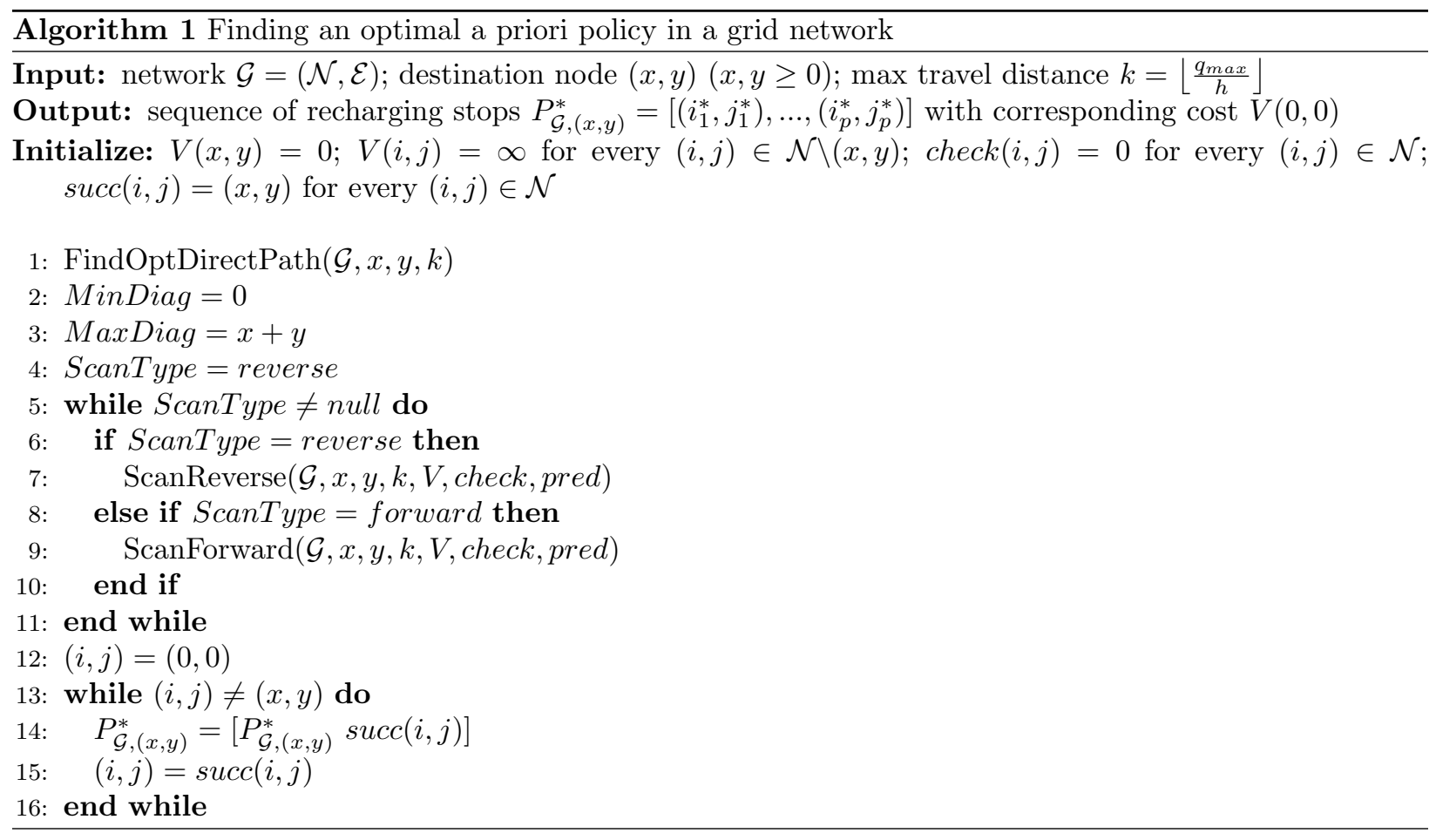

since $x y \leq n=|\mathcal{N}|$, it is $O\left(n^{2}\right)$. In the worst case scenario where the origin and destination are located in the opposite corners of the grid network, the FindOptDirectPath procedure is performed in $O\left(n^{2}\right)$, which is consistent with the earlier found computational complexity of recursive equation (7) for a general network, without the need to run Dijkstra's algorithm to find the optimal path between recharging nodes (as that procedure is trivial in a grid network). However, the FindOptDirectPath procedure is most beneficial for instances where $x y<<|N|$ and $k<<x+y$, or in other words, where the nodes traversed by direct paths constitute a small subset of the entire network node set and the battery size significantly limits the set of nodes that can be considered for the next recharging location.

The Algorithm 1 then searches the network for policies with a lower cost by considering paths with a longer distance but overall lower cost. It carries this out by alternating between the ScanReverse and ScanForward procedures until there are no further value function improvements at any of the network nodes. The bound found by FindOptDirectPath significantly limits the number of nodes that are explored during each scan step and improves the computational efficiency. Starting with the diagonal partition containing the destination node (indexed $x+y$ ), the ScanReverse procedure updates the value function for other nodes along that diagonal, as well as for the nodes on the diagonals with a lower index yet within a distance of $k=\left\lfloor q_{\max } / h\right\rfloor$ from an updated node. The procedure then advances to the diagonal with the next highest 
index, updating nodes along that diagonal as well as along diagonals with lower indexes, and it continues until a diagonal along which no nodes have updated value functions is reached. From this diagonal, the ScanForward procedure operates analogously to ScanReverse but in the forward direction, searching for nodes to update along the same diagonal or along diagonals with a higher index. The final value at the origin upon termination of the algorithm represents the total cost of an optimal a priori policy. (See Appendix A.1 for detailed pseudocodes for procedures FindOptDirectPath, ScanReverse and ScanForward.) We let $B$ correspond to the number of times Algorithm 1 performs the pair of ScanReverse and ScanForward procedures, and the total run time for the algorithm is $O\left(B n^{2}\right)$. Note that we cannot find a proper bound on $B$ (see Section 6.1 .2 for more discussion), and in the worst case, solving recursive equation (7) adapted to a grid network, which can be done in $O\left(n^{2}\right)$, is a better solution approach. However, larger $B$ values correspond to greater deviation from a direct path, and in a realistic setting, drivers are not interested in deviating too far from a direct path on the way to their destination (corresponding to small $B$ ) and $x y<<|N|$. Therefore, in most practical applications, Algorithm 1 delivers significant computational benefit.

The following theorem establishes optimal termination of the algorithm in finite time.

Theorem 10 Algorithm 1 returns an optimal a priori policy in finite time.

Proof. The LB function in ScanReverse and ScanForward procedures of Algorithm 1 (see Appendix A.1) computes the lower bound on the minimum travel time from a given node in the network to the destination node. LB is positive and monotone increasing with respect to the distance between a node and the destination and it restricts the number of nodes to be explored by the algorithm to a finite set. Furthermore, the number of iterations of ScanReverse and ScanForward is finite because of the suboptimality of cycles. Therefore, Algorithm 1 returns an optimal a priori policy in finite time.

Remark 1 It is important to note that Algorithm 1 returns an optimal sequence of recharging locations and corresponding recharge amounts, but not an explicit path. Due to the grid network structure, there is always more than one shortest path between any two distinct nodes, as long as they are not horizontally or vertically aligned, and the routing policy corresponding to the identified optimal charging policy is not unique. Therefore, as long as the EV driver follows any direct path between each pair of consecutive recharge locations, the resulting routing and recharging policy will be optimal for our a priori setting. 


\subsubsection{Illustrative examples of optimal a priori policies}

It is interesting to note that the total number of scan procedures completed (both ScanReverse and ScanForward) in Algorithm 1 for which the objective value continues to improve directly corresponds to the shape of an optimal path. For example, if an optimal policy is obtained immediately after the FindOptDirectPath procedure, then the corresponding path is a direct path between the origin and destination. An improvement to the objective value after one call to the ScanReverse procedure implies that the optimal path is not direct but the sequence of diagonals corresponding to each stop is nondecreasing. Additional improvements after the initial ScanForward procedure indicate that the sequence of diagonals has at least one sign change, with further improvements signaling even more sign changes.

Some numerical examples of optimal a priori policies are illustrated in Figure 6. Here, the white area corresponds to nodes with randomly generated node availability probabilities and expected wait times from uniform distributions $U(0,1)$ and $U(0,240)$, respectively, while shaded areas denotes regions without any charging stations. In the first example (Figure 6(a)), the optimal a priori policy corresponds to a direct path between the origin and destination. This policy is found within the FindOptDirectPath procedure, and no improvements to it are made after calls to the ScanReverse and ScanForward procedures. The second example (Figure 6(b)) illustrates a policy corresponding to an indirect path. Whereas a direct path would have all recharging stops within the bounding box between the origin and destination, the shown path includes stops outside of the bounding box. However, note that the indices of the diagonals corresponding to the sequence of recharging stops are nondecreasing. This implies that the optimal a priori policy is obtained after the first ScanReverse procedure. The third example illustrates an optimal policy that is obtained after multiple calls to the ScanReverse and ScanForward procedures (Figure 6(c)). In this case, the sequence of diagonals corresponding to the recharging stops has two sign changes (i.e., decreases, then increases and decreases again), implying that the policy was obtained after two ScanReverse procedures (and one ScanForward procedure).

\subsection{Heuristics for Adaptive Policies for a Grid Network}

We now describe two heuristic methods for obtaining adaptive policies in a grid network that are based on a priori policies and the solution approach presented in Section 6.1. The first heuristic generates multiple sample paths to quickly estimate the value of adaptive recharging decisions and selects the best path, while the second heuristic captures additional adaptivity with regard to path selection in order to provide further improvement. 


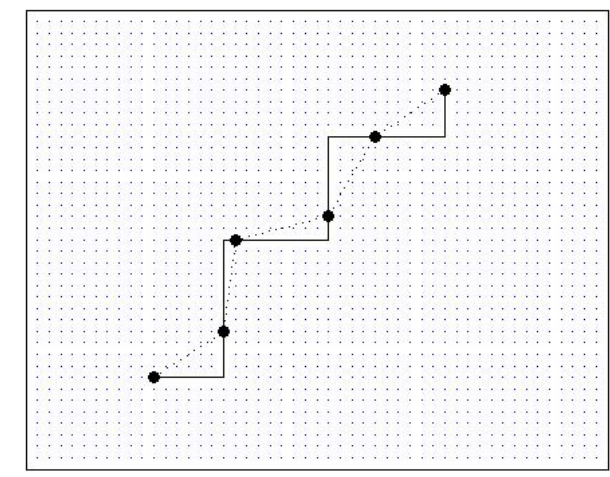

(a) Direct path

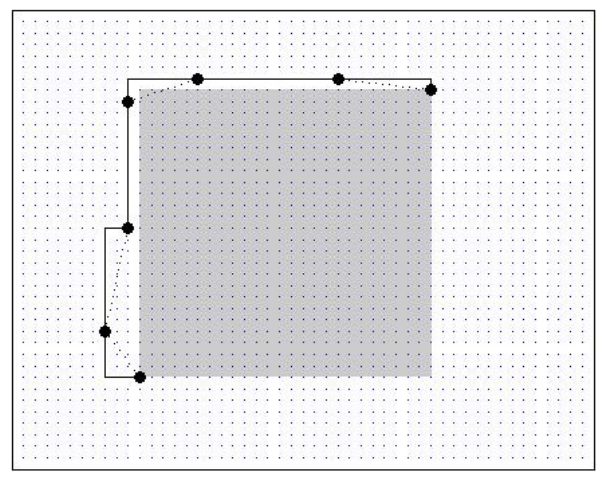

(b) Indirect path (nondecreasing diagonals)

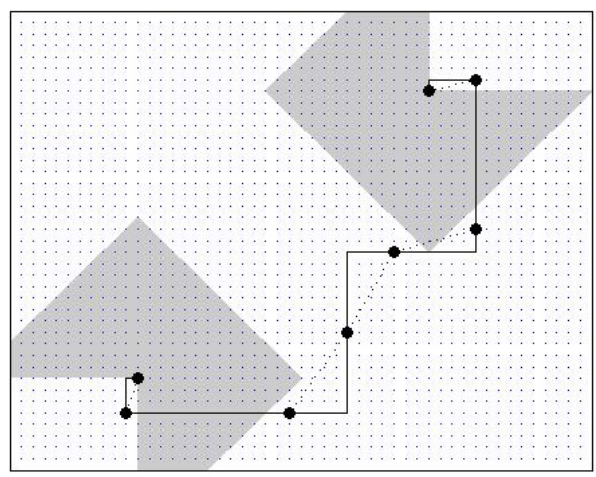

(c) Indirect path (decreasing/increasing diagonals)

Figure 6: Examples of a priori policies in a network. Highlighted nodes represent recharging stops (with a sample path shown as a solid line), and shaded areas represent regions where charging stations are never available.

\subsubsection{Heuristic 1: Adaptive recharging only}

Analogous to the heuristic solution method presented in Section 5.3.1, we use a two-stage solution approach: first selecting a path for the EV to follow, and then selecting an adaptive recharging policy for the fixed path. We solve recursive formulation (4) to "price" each potential path considered by the first-stage problem and bound the set of paths to be considered using the optimal a priori policy found by Algorithm 1. Unlike most of the general network problem instances studies in Section 5, an optimal a priori policy in a grid network does not always uniquely specify a path. Instead, Algorithm 1 only identifies the optimal recharging locations to be used by the EV, and there are numerous paths that can be followed between each pair of consecutive recharging locations (see Remark 1). Since the number of actual paths corresponding to an optimal a priori 
solution can be large, we randomly select only a subset of these paths to be considered in the second stage. That is, in our first heuristic, outlined in Algorithm 2, we construct numPaths different randomly sampled paths that contain the nodes corresponding to the recharging stops in the optimal a priori policy. With the aim to minimize travel and recharging costs, we require that our sampled paths be direct paths between each consecutive pair of a priori stop locations with no detours or cycles. We then calculate the value of an optimal adaptive policy along each path and choose the path with the lowest value.

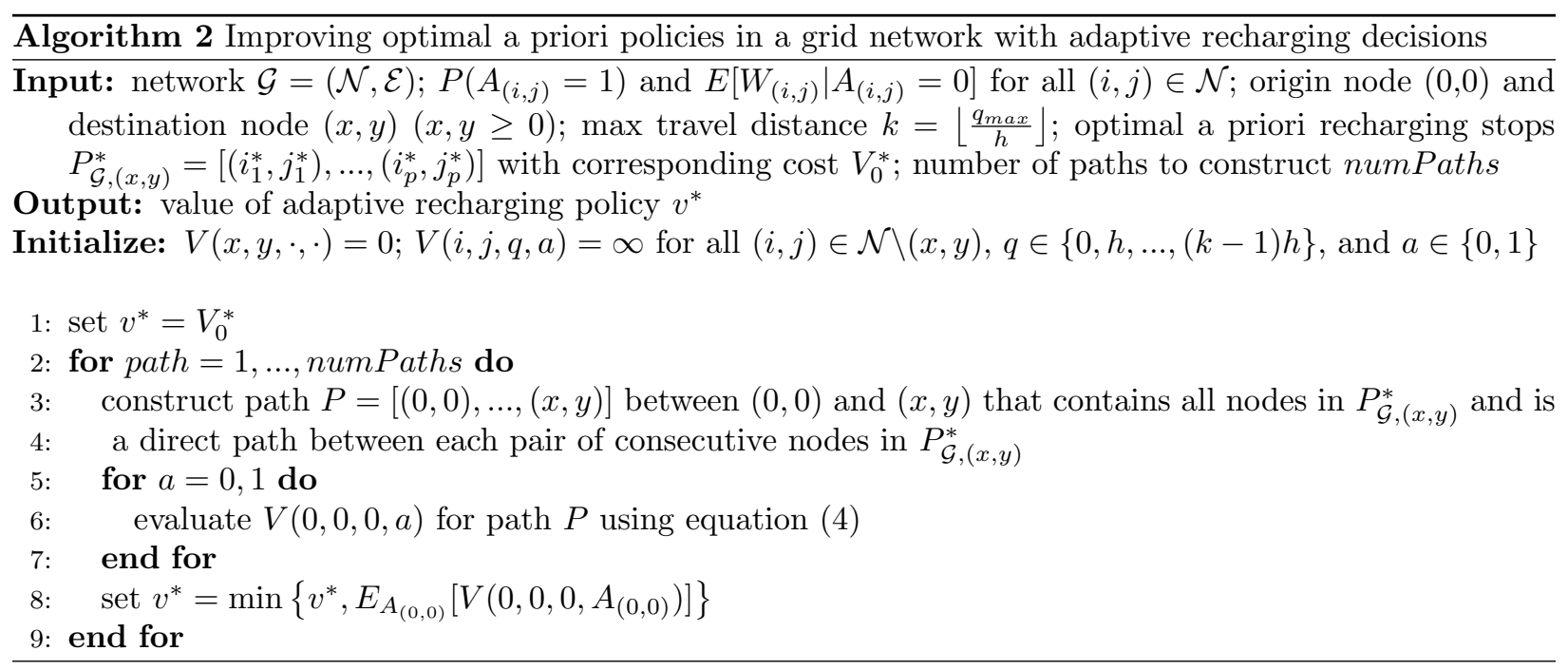

The computational complexity of Heuristic 1 can be analyzed by adding the run time of Algorithm 1 $\left(O\left(B n^{2}\right)\right)$ and Algorithm 2, which is $O\left(\right.$ numPaths $\left.\cdot n^{2}\right)$. Then Heuristic 1 finds adaptive recharging policy for a grid network in $O\left(\max (B\right.$, numPaths $\left.) n^{2}\right)$. Once again, note that in most instances the number of nodes traversed by the considered path between origin and destination is significantly smaller than $|N|$, and the runtime parameter $n$ corresponds to the number of nodes along the path, resulting in a significantly more efficient algorithm than $O\left(\max (B\right.$, numPaths $\left.) n^{2}\right)$ makes it appear.

Because we choose numPaths to be less than the value that would be required for total enumeration over all possible paths, we are not guaranteed to find the path with the lowest optimal value. The runtime of the algorithm increases linearly with the number of paths explored. At the same time, the expected marginal improvement that each additional path delivers diminishes fairly rapidly - a fact supported by our numerical results. The goal of this heuristic is to generate a quick and reasonable policy with adaptive recharging decisions. Our next heuristic incorporates adaptive path selection to provide an improved adaptive policy with a lower cost. 


\subsubsection{Heuristic 2: Adaptive routing and recharging}

In our second heuristic, outlined in Algorithm 3, we include both adaptive routing and recharging decisions. As with Heuristic 1, we require that the vehicle visits (but not necessarily recharge at) every stop location identified by the optimal a priori policy and travels along a direct path between consecutive a priori stop locations. Rather than fix the entire path prior to solving for the optimal policy, however, we allow the vehicle to adjust its trajectory as it travels from the origin $(0,0)$ to the destination $(x, y)$. Working backwards from the destination $(x, y)$, Algorithm 3 finds an optimal policy between consecutive a priori stop locations over all possible states (i.e., not just those for which the vehicle's charge level at a stop location is 0). It considers all direct paths between each pair of nodes and permits adaptive decision making for both routing and recharging. Because this method takes advantage of the grid structure as it searches for an optimal policy, it is very efficient and terminates quicker than Algorithm 2 in many cases, even when numPaths is small.

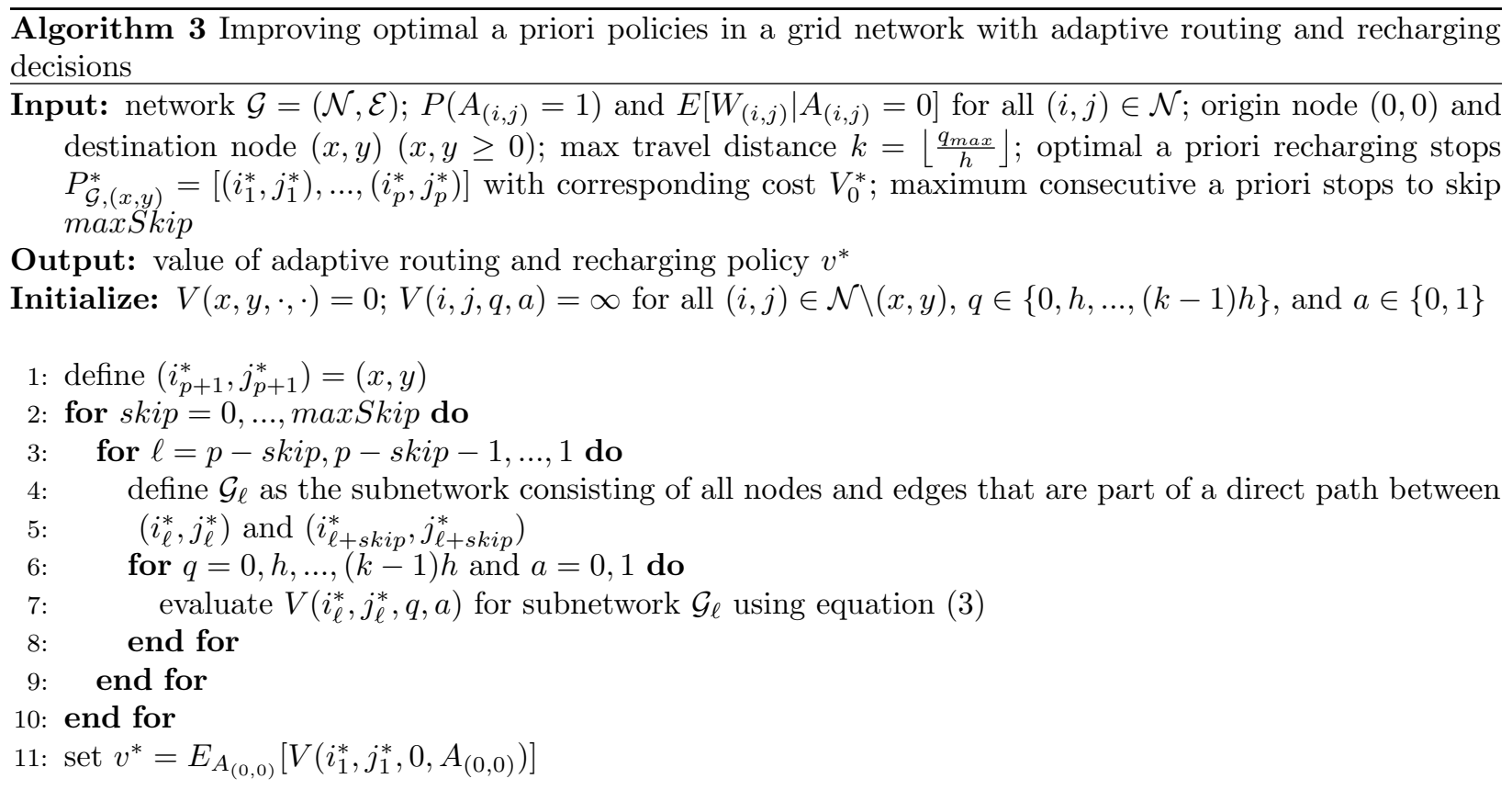

To relax the restriction that every stop location in the optimal a priori policy be visited, the parameter maxSkip can be increased from 0 up to a maximum of $p-1$ (where $p$ is the number of stops in the optimal a priori policy). The value of maxSkip corresponds to the maximum number of consecutive a priori stop locations that may be skipped in order to find an adaptive routing and recharging policy with an even lower cost (see Figure 7). For example, when maxSkip $=0$, every stop location in the optimal a priori policy must be visited (although the vehicle need not stop to recharge at each location). In the case when maxSkip $=2$, 


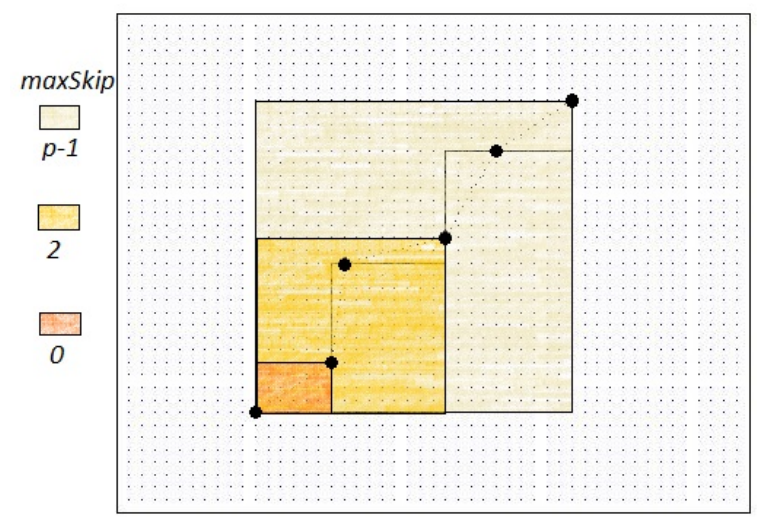

Figure 7: Example of the subsets of network considered by Algorithm 3 for $\max S k i p$ values 0,2 and $p-1$. Highlighting nodes represent recharging stops for optimal a priori policy.

a policy may skip one or two consecutive a priori stop locations at a time, provided that the vehicle follows a direct path between the a priori stop locations it does visit. Then, when $\max S k i p=p-1$, any policy in which the vehicle travels along a direct path between the origin $(0,0)$ and destination $(x, y)$ is permissible. Although the motivating example discussed in Section 3.2 suggests that increasing maxSkip can significantly lower the total cost, our numerical results indicate that in most cases the improvement is negligible and not worth the significant increase in computational time. Therefore, in the next section, we fix maxSkip $=0$ so that only policies that include all a priori stop locations are compared.

\section{$7 \quad$ Numerical results}

We implemented Algorithms 1, 2, and 3 for a variety of simulated settings in order to compare their performance. We use as our network a 500-by-500 node grid with a distance of five miles between adjacent nodes. Expected waiting times and station availabilities at each node are generated from separate uniform distributions as given in Table 1. In Scenario 1, charging stations are available on average half of the time and have expected wait times (if occupied) of around two hours, whereas in Scenario 2, charging stations are more likely to be available but also have longer expected wait times. Thus, the average unconditional expected wait time for a station is similar under each scenario, but the variances are different. For each scenario, we consider a set of 50 random origin-destination pairs separated by distances of between 100 and 300 miles representing trips that an EV driver could reasonably complete in a day, and also another set of 50 random origin-destination pairs with distances ranging from 500 to 1,000 miles that require multiple recharging stops. 


\begin{tabular}{|c|c|c|}
\cline { 2 - 3 } \multicolumn{1}{c|}{} & \multicolumn{2}{c|}{ Distributions } \\
\hline Scenario & $E\left[W_{i, j} \mid A_{i, j}=0\right]$ & $P\left(A_{i, j}=1\right)$ \\
\hline 1 & $U(0,240)$ & $U(0,1)$ \\
2 & $U(240,480)$ & $U(0.8,1)$ \\
\hline
\end{tabular}

Table 1: Distributions of expected wait times (measured in minutes) and availability probabilities at each charging station

We test each algorithm over every scenario and set of trips and for all possible combinations of the parameter settings listed in Table 2. We fix the value for $\alpha$ as 0.8 since that is the most commonly used value in practice [17], and the range of values for $q_{\max }$ corresponds to the maximum driving range of the 2014 Nissan Leaf under different traffic and environmental conditions [8]. For the overcharging cost function, we use

$$
f(z)=2 \cdot(\exp \{z / 5\}-1)
$$

where $z$ is the amount overcharged at a given stop. The expression in (8) captures the real-world dynamics of battery charging and is calibrated based on one driver's experience with overcharging a Leaf [12].

\begin{tabular}{|c|c|c|}
\hline Parameter & Value(s) & Units \\
\hline$\alpha$ & 0.8 & N/A \\
$\gamma$ & 1 & time \\
$h$ & 5 & energy \\
$q_{\max }$ & $\{70,80,90,100\}$ & energy \\
$s$ & $\{1,10,100\}$ & time \\
$t$ & $\{5,10,15\}$ & time \\
numPaths & 5 & N/A \\
maxSkip & 0 & N/A \\
\hline
\end{tabular}

Table 2: Model parameter settings

\subsection{Value of adaptive decision making}

In this section, we measure the value of adaptive decision making by comparing the costs of the policies obtained by the two heuristic methods with that of an optimal a priori policy. Note that the primary benefit of an adaptive policy is a reduction in the total expected waiting time for a given trip. Therefore, rather than compare the aggregate cost savings obtained from Algorithms 2 and 3 to the total cost of a policy found by Algorithm 1, we instead compare them only to the total expected waiting cost of an optimal a priori policy. This provides a better reference for understanding the potential savings that adaptivity allows.

The value of adaptive decision making in the two heuristics is illustrated in Figure 8 for trips of different 
lengths and for both grid scenarios. Because Heuristic 2 allows for greater adaptivity than Heuristic 1, it always provides a higher benefit. The benefit is not always so pronounced, however, since the system is ergodic and the vehicle still must visit all of the a priori stop locations (because maxSkip $=0$ ), and thus the ability to explore alternative paths is limited. In addition, the value of adaptivity is greater for longer trips and for Scenario 2. Long trips have more opportunities for the vehicle to find available charging stations without having to wait, and there is also greater flexibility with regard to path selection due to the higher number of nodes between the origin and destination. Comparing the two grid scenarios, the value of adaptivity is higher in Scenario 2 because the expected wait times at unavailable charging stations are longer than in Scenario 1 despite the unconditional expected wait times $\left(E\left[W_{i}\right]\right)$ being similar. The vehicle is more likely to encounter an available charging station before running out of charge and being forced to stop at a station regardless of its availability, whereas in Scenario 1 there is less incentive to recharge earlier than necessary.
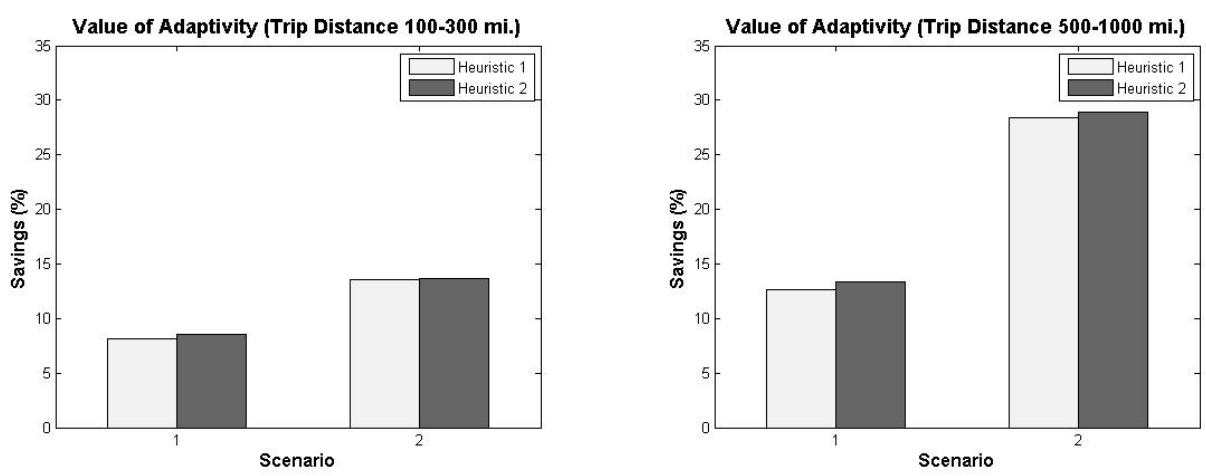

Figure 8: Value of adaptive decision making as a percentage of the total expected waiting time in an optimal a priori policy

\subsection{Number of stops comparison}

As a result of the vehicle being able to make adaptive recharging decisions and take advantage of opportunities to recharge at available charging stations, the stopping locations tend to occur sooner along the route than in the optimal a priori policy. When this effect is compounded over the length of the entire path, it can lead to instances in which the number of stops in an adaptive policy is greater than the number of stops in an a priori policy. Figure 9 shows the additional number of stops in the heuristic policies relative to that of the optimal a priori policy. Understandably, the longer trips tend to have a greater number of stops when adaptive decisions are permitted since the combined effect of recharging earlier than necessary is greater and 
is more likely to lead to an extra stop.
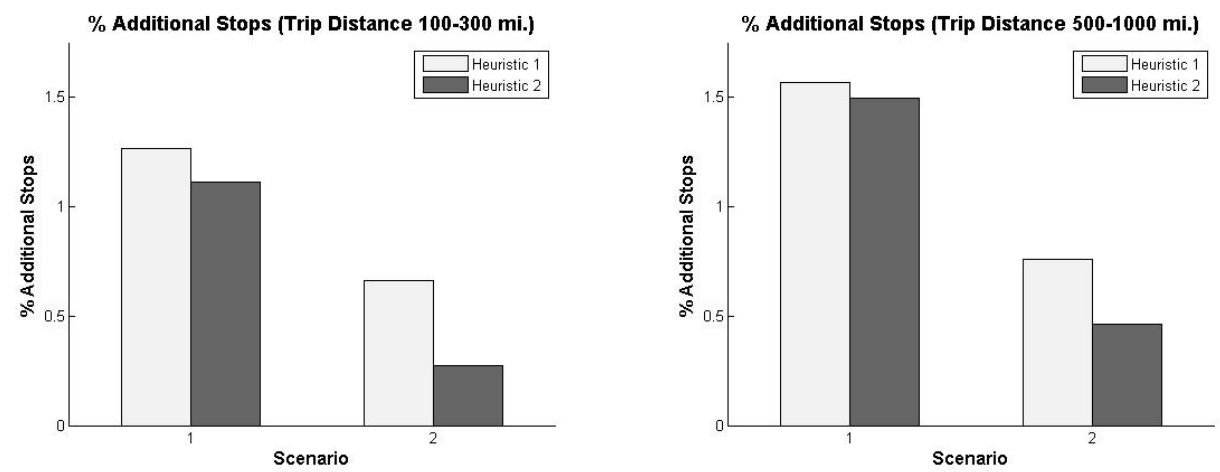

Figure 9: Average difference in number of stops between heuristic and optimal a priori policies

Averaged over all instances, the net additional increase in the number of stops is less than $2 \%$. However, it can be seen that the effect is greater for Scenario 1 than for Scenario 2, which implies that the adaptive policies are more likely to stop to recharge if a charging station is available. This is due to the fact that the average probability of a charging station being available is lower in Scenario 1 than in Scenario 2, and thus the vehicle is more likely to charge sooner than necessary to avoid the chance of having to stop at an unavailable station. Heuristic 1 also tends to have more stops than Heuristic 2 because when the path is fixed, there are fewer stations from which to choose in order to delay recharging. With adaptive routing, the vehicle has several options and can pick the path that offers the greatest opportunity to delay recharging and reduce the likelihood of having to make an additional stop later along its route. Such opportunities are more prevalent in Scenario 2 than in Scenario 1, as the mean station availability is higher, and thus the difference in the number of stops between the two heuristics is greater in Scenario 2.

\section{Conclusion}

In this paper, we explore the problem of finding an optimal routing and recharging policy in a network with uncertain charging station availability. We begin with an adaptive recharging problem on a fixed path and then generalize the setting to include routing decisions on a general network. In the network setting, we identify properties of optimal policies and evaluate computation complexity of the problem. We use these properties to develop efficient algorithms for finding an optimal a priori policy and then present solution approaches to an adaptive problem that build on a priori policy. We further enhance our solution approaches to a special case of grid network. We present and implement two heuristics for obtaining an adaptive policy 
for a grid network, and we conduct detailed numerical experiments to evaluate the performance of our solution methods and estimate the value of adaptivity.

The uncertainty of charging station availability within the network and the ability of the vehicle to adaptively choose which route to follow and where to recharge are unique and important features of our model. Our inclusion of a convex recharging cost function further improves the suitability of this work for capturing the costs incurred by EV drivers. As long as charging stations remain scarce and range anxiety persists, it is critical for EV drivers to be equipped with the necessary decision-support tools to minimize their time spent waiting for charging stations to become available and also make optimal routing and recharging decisions.

As part of our future work, we plan to further explore the value of adaptivity. We will refine our solution methods to identify additional opportunities within a network setting for an EV driver to benefit from adaptive decision making. We also intend to determine the value of an EV driver knowing not only the availability of the charging station at his or her current location, but also the availability of other nearby charging stations. Having such online information could potentially be of great benefit by enabling the driver to anticipate the likelihood of distant charging stations being available and plan his or her route accordingly. However, the exponential increase in computational time required to incorporate distant stations into a driver's decisions would need to be addressed. 


\section{A Appendix}

\section{A.1 Code for procedures used in Algorithm 1}

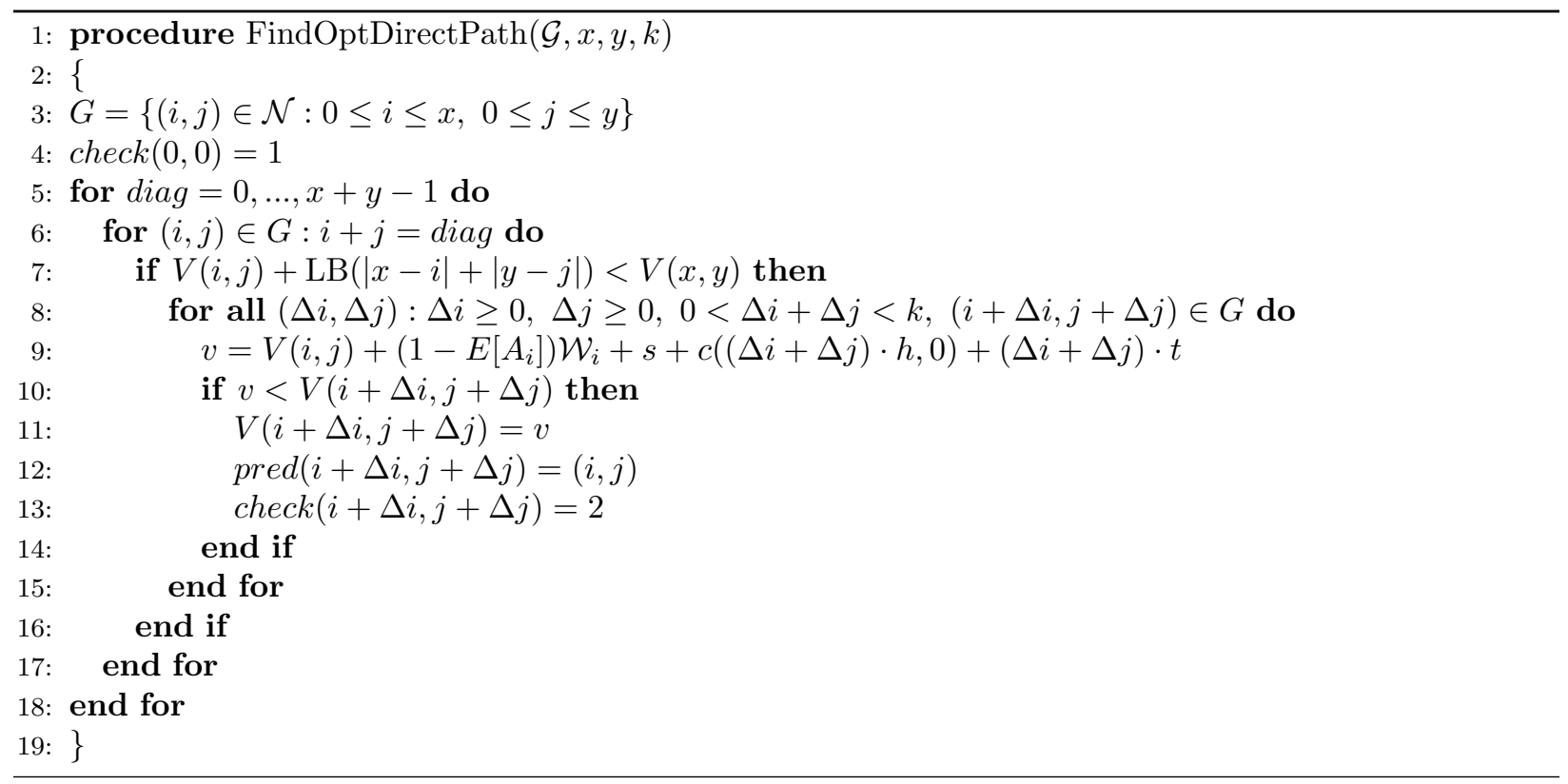




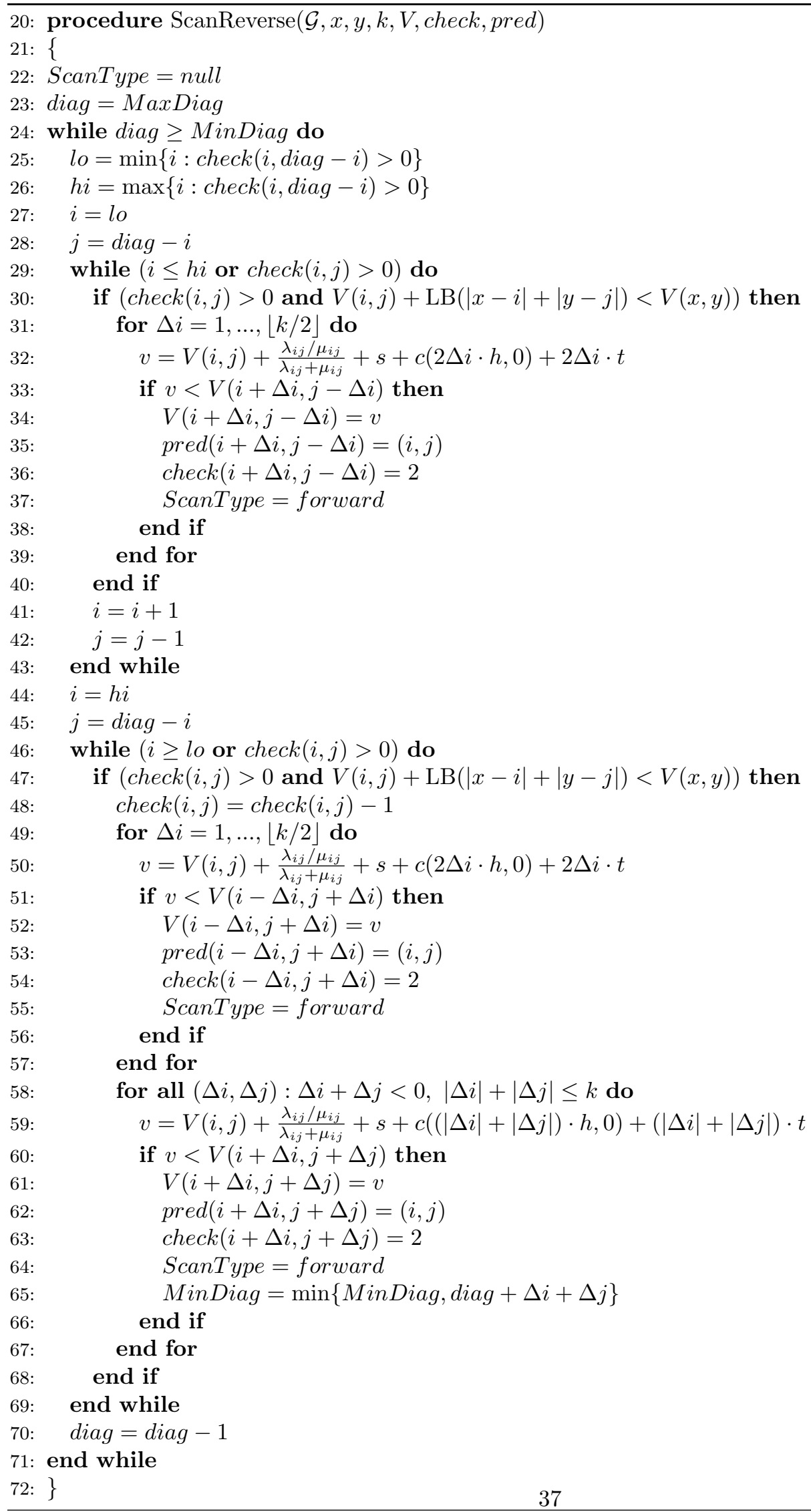


73: procedure $\operatorname{ScanForward}(\mathcal{G}, x, y, k, V$, check, pred $)$

74: \{

75: ScanType $=$ null

76: $\operatorname{diag}=$ MinDiag

77: while diag $\leq$ MaxDiag do

78: $\quad l o=\min \{i: \operatorname{check}(i, \operatorname{diag}-i)>0\}$

79: $\quad h i=\max \{i: \operatorname{check}(i, \operatorname{diag}-i)>0\}$

80: $\quad i=l o$

81: $j=\operatorname{diag}-i$

82: $\quad$ while $(i \leq h i$ or $\operatorname{check}(i, j)>0)$ do

83: $\quad$ if $(\operatorname{check}(i, j)>0$ and $V(i, j)+\mathrm{LB}(|x-i|+|y-j|)<V(x, y))$ then

84: $\quad$ for $\Delta i=1, \ldots,\lfloor k / 2\rfloor$ do

85:

86:

87:

88:

89:

90:

91:

92:

93:

94:

95:

96:

97:

98:

99:

100:

101:

102:

103:

104:

105:

106:

107:

108:

109:

110:

111:

112:

113:

114:

115:

116 :

$117:$

118:

119:

120:

121:

122 :

123:

124: 


\section{A.2 Omitted Proofs}

\section{A.2.1 Proof of Lemma 1}

Proof. Suppose $\pi_{\mathcal{N}}^{R}: \mathcal{S} \rightarrow \mathcal{A}^{R}$ is a feasible recharging policy, and there exists a state $\left(i, q_{i}, a_{i}\right) \in \mathcal{S}$ such that $\pi_{\mathcal{N}}^{R}\left(i, q_{i}, a_{i}\right)=\sum_{\ell=i}^{k-1} h_{\ell}-q_{i}+\Delta>0$ for some $k \in(i+1, \ldots, n-1)$ and $0 \leq \Delta<h_{k}$. Then consider an alternate policy $\pi_{\mathcal{N}}^{R^{\prime}}: \mathcal{S} \rightarrow \mathcal{A}^{R}$ where

$$
\pi_{\mathcal{N}}^{R^{\prime}}\left(j, q_{j}, a_{j}\right)= \begin{cases}\pi_{\mathcal{N}}^{R}\left(j, q_{j}, a_{j}\right)-\Delta, & \left(j, q_{j}, a_{j}\right)=\left(i, q_{i}, a_{i}\right) \\ \pi_{\mathcal{N}}^{R}\left(j, q_{j}+\Delta, a_{j}\right)+\Delta I_{\pi_{\mathcal{N}}^{R}\left(j, q_{j}+\Delta, a_{j}\right)>0}, & j \in(i+1, \ldots, k) \text { and } q_{j}=\sum_{\ell=j}^{k-1} h_{\ell} \\ \pi_{\mathcal{N}}^{R}\left(j, q_{j}, a_{j}\right), & \text { otherwise }\end{cases}
$$

for any $\left(j, q_{j}, a_{j}\right) \in \mathcal{S}$, where $I_{\pi_{\mathcal{N}}^{R}\left(j, q_{j}+\Delta, a_{j}\right)>0}$ equals 1 if $\pi_{\mathcal{N}}^{R}\left(j, q_{j}+\Delta, a_{j}\right)>0$ and 0 otherwise. In this alternate policy, the vehicle's charge level when departing from node $i$ is $\sum_{\ell=i}^{k-1} h_{\ell}$ instead of $\sum_{\ell=i}^{k-1} h_{\ell}+\Delta$. The next node where the vehicle stops to recharge is the same as in $\pi_{\mathcal{N}}^{R}$, although the vehicle recharges an extra amount $\Delta$ so that the resulting charge level is the same as in the original policy. Beyond that node, both policies are identical.

Suppose that $j$ is the next node after $i$ at which the vehicle stops to recharge for a given sequence $\left(a_{i+1}, \ldots, a_{j}\right)$ of observed station availabilities (i.e., $A_{\ell}=a_{\ell}$ for all $\left.\ell \in(i+1, \ldots, j)\right)$. Then the expected cost of the policy $\pi_{\mathcal{N}}^{R}$ for the state $\left(i, q_{i}, a_{i}\right)$ is

$$
\begin{aligned}
C_{\pi_{\mathcal{N}}^{R}}\left(i, q_{i}, a_{i}\right) \mid\left(A_{\ell}=a_{\ell} \forall \ell \in(i+1, \ldots, j)\right)= & \sum_{\ell=i}^{j-1} t_{\ell}+s+\left(1-a_{i}\right) \mathcal{W}_{i}+c\left(\sum_{\ell=i}^{k-1} h_{\ell}-q_{i}+\Delta, q_{i}\right)+ \\
& C_{\pi_{\mathcal{N}}^{R}}\left(j, \sum_{\ell=j}^{k-1} h_{\ell}+\Delta, a_{j}\right) .
\end{aligned}
$$

Now suppose that the same sequence of station availabilities is observed under policy $\pi_{\mathcal{N}}^{R}$. Then the expected cost of the policy under such a scenario is

$$
\begin{aligned}
C_{\pi_{\mathcal{N}}^{R^{\prime}}}\left(i, q_{i}, a_{i}\right) \mid\left(A_{\ell}=a_{\ell} \forall \ell \in(i+1, \ldots, j)\right)= & \sum_{\ell=i}^{j-1} t_{\ell}+s+\left(1-a_{i}\right) \mathcal{W}_{i}+c\left(\sum_{\ell=i}^{k-1} h_{\ell}-q_{i}, q_{i}\right)+ \\
& C_{\pi_{\mathcal{N}}^{R^{\prime}}}\left(j, \sum_{\ell=j}^{k-1} h_{\ell}, a_{j}\right) .
\end{aligned}
$$

When we compare the costs of the two policies for the given realization of observed station availabilities, we 
find that

$$
\begin{aligned}
& (9)-(10)=c\left(\sum_{\ell=i}^{k-1} h_{\ell}-q_{i}+\Delta, q_{i}\right)+C_{\pi_{\mathcal{N}}^{R}}\left(j, \sum_{\ell=j}^{k-1} h_{\ell}+\Delta, a_{j}\right)-c\left(\sum_{\ell=i}^{k-1} h_{\ell}-q_{i}, q_{i}\right)-C_{\pi_{\mathcal{N}}^{R \prime}}\left(j, \sum_{\ell=j}^{k-1} h_{\ell}, a_{j}\right) \\
& =c\left(\Delta, \sum_{\ell=i}^{k-1} h_{\ell}\right)+C_{\pi_{\mathcal{N}}^{R}}\left(j, \sum_{\ell=j}^{k-1} h_{\ell}+\Delta, a_{j}\right)-C_{\pi_{\mathcal{N}}^{R^{\prime}}}\left(j, \sum_{\ell=j}^{k-1} h_{\ell}, a_{j}\right) \\
& =c\left(\Delta, \sum_{\ell=i}^{k-1} h_{\ell}\right)+c\left(\pi_{\mathcal{N}}^{R}\left(j, \sum_{\ell=j}^{k-1} h_{\ell}+\Delta, a_{j}\right), \sum_{\ell=j}^{k-1} h_{\ell}+\Delta\right)-c\left(\pi_{\mathcal{N}}^{R^{\prime}}\left(j, \sum_{\ell=j}^{k-1} h_{\ell}, a_{j}\right), \sum_{\ell=j}^{k-1} h_{\ell}\right) \\
& =c\left(\Delta, \sum_{\ell=i}^{k-1} h_{\ell}\right)+c\left(\pi_{\mathcal{N}}^{R}\left(j, \sum_{\ell=j}^{k-1} h_{\ell}+\Delta, a_{j}\right), \sum_{\ell=j}^{k-1} h_{\ell}+\Delta\right)-c\left(\pi_{\mathcal{N}}^{R}\left(j, \sum_{\ell=j}^{k-1} h_{\ell}+\Delta, a_{j}\right)+\Delta, \sum_{\ell=j}^{k-1} h_{\ell}\right) \\
& =c\left(\Delta, \sum_{\ell=i}^{k-1} h_{\ell}\right)-c\left(\Delta, \sum_{\ell=j}^{k-1} h_{\ell}\right) \\
& =\left(\gamma \Delta+f\left(\left(\sum_{\ell=i}^{k-1} h_{\ell}+\Delta-\alpha q_{\max }\right)^{+}\right)-f\left(\left(\sum_{\ell=i}^{k-1} h_{\ell}-\alpha q_{\max }\right)^{+}\right)\right)- \\
& \left(\gamma \Delta+f\left(\left(\sum_{\ell=j}^{k-1} h_{\ell}+\Delta-\alpha q_{\max }\right)^{+}\right)-f\left(\left(\sum_{\ell=j}^{k-1} h_{\ell}-\alpha q_{\max }\right)^{+}\right)\right) \\
& \left.\geq \nabla f\left(\left(\sum_{\ell=i}^{k-1} h_{\ell}-\alpha q_{\max }\right)^{+}\right) \Delta-\nabla f\left(\left(\sum_{\ell=j}^{k-1} h_{\ell}+\Delta-\alpha q_{\max }\right)^{+}\right) \Delta \quad \text { (by convexity of } f(\cdot)\right) \\
& \geq 0
\end{aligned}
$$

since $\sum_{\ell=i}^{k-1} h_{\ell}>\sum_{\ell=j}^{k-1} h_{\ell}+\Delta$. The expression in (11) holds for any given sequence $\left(a_{i+1}, \ldots, a_{j}\right)$ of observed station availabilities such that $j$ is the next node after $i$ where the vehicle recharges, and because the probability of the sequence occurring is the same under both policies, the (unconditional) expected cost of the policy $\pi_{\mathcal{N}}^{R^{\prime}}$ is no greater than that of $\pi_{\mathcal{N}}^{R}$ for the state $\left(i, q_{i}, a_{i}\right)$. It follows that there exists an optimal recharging policy $\pi_{\mathcal{N}}^{R}: \mathcal{S} \rightarrow \mathcal{A}^{R}$ such that

$$
\pi_{\mathcal{N}}^{R *}\left(i, q_{i}, a_{i}\right) \in\left\{\left(\sum_{\ell=i}^{k-1} h_{\ell}-q_{i}\right)^{+}: k \in(i+1, \ldots, n-1), \sum_{\ell=i}^{k-1} h_{\ell} \leq q_{\max }\right\}
$$

for all $\left(i, q_{i}, a_{i}\right) \in \mathcal{S}$. 


\section{A.2.2 Proof of Lemma 2}

Proof. We prove the claim by induction. At node 0 , we have $q_{0}=0 \in \mathcal{H}(1)$, and by Lemma 1 , there exists an optimal recharging policy $\pi_{\mathcal{N}}^{R^{*}}$ such that $\pi_{\mathcal{N}}^{R^{*}}(0,0, \cdot) \in \mathcal{H}(1)$.

Then assuming the claim holds for a node $i \in(0, \ldots, n-2)$, suppose $q_{i}=\sum_{\ell=i}^{j-1} h_{\ell} \in \mathcal{H}(i)$ for some $j \in(i, \ldots, n-1)$ and $\pi_{\mathcal{N}}^{R *}\left(i, q_{i}, a_{i}\right)=\sum_{\ell=j}^{j^{\prime}-1} h_{\ell} \in \mathcal{H}(j)$ for some $j^{\prime} \in(j, \ldots, n-1)$. At node $i+1$, the vehicle's charge level is

$$
q_{i+1}=\sum_{\ell=i}^{j-1} h_{\ell}+\sum_{\ell=j}^{j^{\prime}-1} h_{\ell}-h_{i}=\sum_{\ell=i+1}^{j^{\prime}-1} h_{\ell} \in \mathcal{H}(i+1),
$$

and by Lemma 1, we have

$$
\begin{aligned}
\pi_{\mathcal{N}}^{R^{*}}\left(i+1, q_{i+1}, \cdot\right) & \in\left\{\left(\sum_{\ell=i+1}^{k-1} h_{\ell}-q_{i+1}\right)^{+}: k \in(i+2, \ldots, n-1), \sum_{\ell=i}^{k-1} h_{\ell} \leq q_{\max }\right\} \\
& \subseteq\left\{\sum_{\ell=j^{\prime}}^{k-1} h_{\ell}: k \in\left(j^{\prime}, \ldots, n-1\right), \sum_{\ell=i}^{k-1} h_{\ell} \leq q_{\max }\right\} \\
& \subseteq H\left(j^{\prime}\right) .
\end{aligned}
$$

Thus, the claim holds for node $i+1$ as well, and it follows that $q_{i} \in \mathcal{H}(i)$ and $\pi_{\mathcal{N}}^{R^{*}}\left(i, q_{i}, a_{i}\right) \in \mathcal{H}(k)$ for any realized state $\left(i, q_{i}, a_{i}\right) \in \mathcal{S}$ given that the initial state is $(0,0, \cdot)$. 


\section{References}

[1] Artmeier, A., Haselmayr, J., Leucker, M., \& Sachenbacher, M. (2010). The shortest path problem revisited: Optimal routing for electric vehicles. Lecture Notes in Computer Science, 6359, 309-316.

[2] Bakker, J. J. (2011). Contesting range anxiety: The role of electric vehicle charging infrastructure in the transportation transition. Master's thesis, Eindhoven University of Technology.

[3] Dijkstra, E. W. (1959). A note on two problems in connexion with graphs. Numerische Mathematik, 1, 269271.

[4] Eisner, J., Funke, S., \& Storandt, S. (2011). Optimal route planning for electric vehicles in large networks. Proceedings of the 25th Association for the Advancement of Artificial Intelligence Conference.

[5] Electric vehicle charging station locations. (2014). U.S. Department of Energy, Office of Efficiency and Renewable Energy, Alternative Fuels Data Center. Retrieved from http://www.afdc.energy.gov/fuels/electricity_locations.html

[6] Ferrucci, F. (2013). Pro-active Dynamic Vehicle Routing: Real-time Control and Request-forecasting Approaches to Improve Customer Service. Physica-Verlag.

[7] Florian, M., Lenstra, J. K., \& Rinnooy Kan, A. H. G. (1980). Deterministic production planning: Algorithms and complexity. Management Science, 26, 669-679.

[8] Fuel Economy Guide. (2014). U.S. Department of Energy, Office of Energy Efficiency and Renewable Energy, and U.S. Environmental Protection Agency. DOE/EE-0957. Retrieved from http://www.fueleconomy.gov/feg/pdfs/guides/FEG2014.pdf

[9] Khuller, S., Malekian, A., \& Mestre, J. (2007). To fill or not to fill: The gas station problem. Proceedings of the 15th Annual European Symposium on Algorithms, 4698, 534-545.

[10] Klabjan, D. \& Sweda, T. (2011). The nascent industry of electric vehicles. Wiley Encyclopedia of Operations Research and Management Science.

[11] Klampfl, E., Gusikhin, O., Theisen, K., Liu, Y., \& Giuli, T. J. (2008). Intelligent refueling advisory system. White paper.

[12] Laur, D. (2013, April 10). Fast charging to 100\%. My Nissan Leaf Forum. Retrieved from http: //www . mynissanleaf . com/viewtopic $\cdot$ php?f=27\&t=12263\&start=10\#p282113 
[13] Lin, S. H., Gertsch, N., \& Russell, J. R. (2007). A linear-time algorithm for finding optimal vehicle refueling policies. Operations Research Letters, 35, 290-296.

[14] Lin, S. H. (2008). Finding optimal refueling policies: A dynamic programming approach. Journal of Computing Sciences in Colleges, 23, 272-279.

[15] Lin, S. H. (2008). Finding optimal refueling policies in transportation networks. Proceedings of the 4th International Conference on Algorithmic Aspects in Information and Management, 5034, 280-291.

[16] Millner, A. (2010). Modeling lithium ion battery degradation in electric vehicles. Proceedings of the Conference on Innovative Technologies for an Efficient and Reliable Electricity Supply, 349-356.

[17] Nissan Leaf Digital Brochure. (2014). Nissan USA. Retrieved from http://www.nissanusa.com/content/dam/nissan/ request-brochure/en/2014/pdf/2014-nissan-leaf-en.pdf

[18] Powell, W. B. (2007). Approximate Dynamic Programming: Solving the Curses of Dimensionality, Wiley Series in Probability and Statistics, Wiley-Interscience.

[19] Sachenbacher, M., Leucker, M., Artmeier, A., \& Haselmayr, J. (2011). Efficient energy-optimal routing for electric vehicles. Proceedings of the 25th Association for the Advancement of Artificial Intelligence Conference.

[20] Serrao, L., Onori, S., Sciarretta, A., Guezennec, Y., \& Rizzoni, G. (2011). Optimal energy management of hybrid electric vehicles including battery aging. Proceedings of American Control Conference, 21252130.

[21] Sioshansi, R. \& Denholm, P. (2009). Emissions impacts and benefits of plug-in hybrid electric vehicles and vehicle-to-grid services. Environmental Science and Technology, 43, 1199-1204.

[22] Sovacool, B. K. \& Hirsh, R. F. (2009). Beyond batteries: An examination of the benefits and barriers to plug-in hybrid electric vehicles (PHEVs) and a vehicle-to-grid (V2G) transition. Energy Policy, 37, 1095-1103.

[23] Suzuki, Y. (2008). A generic model of motor-carrier fuel optimization. Naval Research Logistics, 55, 737-746.

[24] Suzuki, Y. (2009). A decision support system of dynamic vehicle refueling. Decision Support Systems, $46,522-531$. 
[25] Suzuki, Y., \& Dai, J. (2013). Decision support system of truck routing and refueling: A dual-objective approach. Decision Sciences, 44, 817-842.

[26] Sweda, T. M. \& Klabjan, D. (2012). Finding minimum-cost paths for electric vehicles. Proceedings of the 1st IEEE International Electric Vehicle Conference, 1-4.

[27] Sweda, T. M., Dolinskaya, I. S., \& Klabjan, D. (2014). Optimal recharging policies for electric vehicles. Working Paper 14-01, Department of Industrial Engineering and Management Sciences, Northwestern University.

[28] Thomas, B. W., \& White III, C. C. (2004). Anticipatory route selection. Transportation Science, 38, 473-487.

[29] Thomas, B. W., \& White III, C. C. (2007). The dynamic shortest path problem with anticipation. European Journal of Operations Research, 176, 836-854.

[30] Yen, J. Y. (1971). Finding the $K$ shortest loopless paths in a network. Management Science, 17, 712-716. 